\title{
Localized Surface Plasmon Resonances: Noble Metal Nanoparticle Interaction with Rare-Earth Ions
}

\author{
V.A.G. Rivera, F.A. Ferri and E. Marega Jr. \\ Additional information is available at the end of the chapter
}

http://dx.doi.org/10.5772/50753

\section{Introduction}

Particles of sizes between 1 and $100 \mathrm{~nm}$ show fascinating properties with unusual characteristics that lead to the formation of unique properties in nanosystems, which are not observed in ordinary materials. These are considered hereby as nanoparticles (NPs). Additionally, metallic NPs with sizes smaller than the wavelength of light show strong dipolar excitations in the form of localized surface plasmon resonances (LSPR). LSPRs are nonpropagating excitations of the conduction electrons of metallic NPs coupled to the electromagnetic field [1]. This effect has been the subject of extensive research, both fundamental and with a view to applications [2,3]. The resonance frequency of the oscillation, i.e., the surface plasmon (SP) energy, it is essentially determined by the dielectric properties of the metal and the surrounding medium, and by the particle size and shape. The collective charge oscillation causes a large resonant enhancement of the local field inside aand near the NP. This field enhancement is used in surface-enhanced Raman scattering (SERS) [4] and is currently discussed for potential applications in nonlinear optical devices [5], in optical tweezers [6], and generally for the manipulation of the local photonic density of states.

Rare-earth (RE) elements are a group of chemical elements known as Lanthanides that occur together in the periodic table. These elements are used in common consumer goods such as: computer memory, DVD's, rechargeable batteries, cell phones, car catalytic converters, magnets, fluorescent lighting and much more. Furthermore, play an essential role in modern national defense, e.g.: lanthanum in night-vision goggles, neodymium in laser range-finders, guidance systems, communications; europium in fluorescents and phosphors in lamps and monitors; erbium amplifiers in fiber-optics data transmission; samarium in permanent magnets stable at high temperatures and, others technological applications. We center our 
attention in the RE ions and their optical properties that are widely employed in photonic. Devices of general interest span RE ions concentrations of tens to several thousand parts per million (ppm), resulting in devices of one to tens of meters long such as channel waveguides and optical fiber, respectively. In optical devices, the RE should ideally be confined as a delta function in the center of the core for maximum gain per unit pump power. Thereby, there is a necessary tradeoff between the confinement and the RE ions concentration, such that the more confined structures require a higher rare-earth ions concentration. An important feature that distinguishes them from other optically active ions: they emit and absorb over narrow wavelength ranges, the wavelengths of the emission and absorption transitions depend the host material, the intensities of these transitions are weak, the lifetimes of metastable states are long, and the quantum efficiencies tend to be high, except in aqueous solutions. These properties lead to excellent performance of RE ions in many optical applications. Devices that provide gain (e.g. lasers and amplifiers), must have low scattering losses, and one is restricted to using single-crystal or glass hosts. Whereas in many applications crystalline materials are preferred for reasons that include higher peak cross sections or better thermal conductivities, the versatility of glasses and the broader emission and absorption spectra they provide have led to the use of RE doped glasses in many applications, eventually running into the clustering limit for the particular host glass composition [7].

Most of existing and potential future applications of NPs suffer from damping caused by metal absorption. Sudarkin and Demkovich [8] suggested increasing the propagation length of the surface plasmon polariton ${ }^{1}$ (SPP) by creating the population inversion in the dielectric medium adjacent to the metallic surface (film). Recently, gain-assisted propagation of the SPPs at the interface between a metal and a dielectric with optical gain has been analyzed theoretically $[9,10]$. Thus, NPs embedded in a gain media represent a field enhancement sustained that resonant excitation can lead to a reduction in the threshold for achieving inversion in the optically active surrounding medium, and the presence of gain can counteract the inherent absorption losses in the NP [1]. While this enhancement of plasmon resonances in gain medium not is experimentally confirmed, amplification of fluorescence due to field enhancement in gain medium with metal NPs has been observed [11,12,13,14].

In the present chapter, we explore the physics of localized surface plasmons by first considering the interaction of metal NPs with an electromagnetic wave in order to arrive at the resonance condition (LSPR). After, we show studies of plasmon resonances in particles with different shapes and sizes, and the effects of interactions between particles in the ensemble. Then we will focus on the RE ions, discussing the optical properties of the trivalent ions through the principles of quantum mechanics (in terms of oscillator strengths) with special interest in the interactions between the $4 \mathrm{f}$ electrons themselves. Since all the other electronic shells are spherically symmetric. Finally we present diverse experimental results of the interaction of RE ions interaction with NPs, resulting in an enhancement of the

\footnotetext{
${ }^{1}$ A SPP is an electromagnetic wave propagating along the interface between two media possessing permittivities with opposite signs, such as the metal-dielectric interface.
} 
intensity emission of the RE ions due to long-range electromagnetic interaction between LSPR and the RE ions.

In this context, it is worth mentioning that the main purpose of this chapter is to show some of concrete concept of noble metallic nanoparticle interaction with rare-earth ions exhibiting a field enhancement in transparent medium with noble metal NPs embedded via a localized surface plasmon resonance.

\section{Localized surface plasmon resonance and metallic nanoparticles}

First, we know that a surface plasmon resonance (SPR) can be described as the resonance collective oscillation of valence electrons in a solid stimulated by beam of light incident (electromagnetic field - see Figure 1). The resonance condition is established when the frequency of light matches the natural frequency of valence electrons oscillating against from this restoring force. SPR in nanometer-sized structures is called LSPR. For gold and silver NPs, the resonance falls into the visible region of the electromagnetic spectrum. A striking consequence of this is the bright colors exhibited by particles both in transmitted and reflected light, due to resonantly enhanced absorption and scattering. This effect has found applications for many hundreds of years, for example in the staining of glass for windows or ornamental cups².
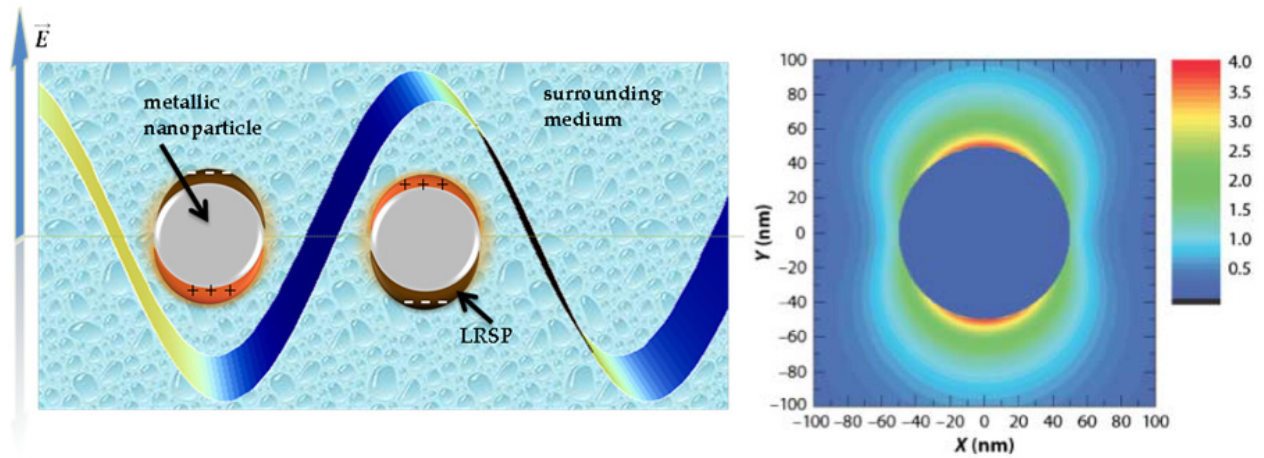

Figure 1. Left: Schematic of the electron charge displacement (valence electrons) in a metallic NP (LSPR) interacting with an incident plane wave, with electric field polarized $E$ into a host matrix. Right: The corresponding electric field strength pattern for a $100 \mathrm{~nm}$ silver sphere, irradiated at a wavelength of $514 \mathrm{~nm}$, this is reproduced from X. Lu et al. [15].

\subsection{Noble metal nanoparticles in an electric field}

For spherical NPs smaller than compared to local variations of the involved electromagnetic fields $(d<<)$ in the surrounding medium, the quasi-static approximation [16], we lead to a

\footnotetext{
${ }^{2}$ Localized surface plasmons have been observed since the Romans who used gold and silver nanoparticles to create colored glass objects such as the Lycurgus Cup (4th Century A.D.). A gold sol in the British museum, created by Michael Faraday in 1857, is still exhibiting its red color due to the plasmon resonance at ,530 nm [L. M. Liz-Marzan, Mater. Today 7, 26 (2004).].
} 
good agreement between theory and experiment. So, one can calculate the spatial field distribution (assuming as a problem of a particle in an electrostatic field), from the Laplace equation for the potential $\nabla^{2} \Phi=0$, therefore the electric field $E=-\nabla \Phi$. Besides, the harmonic time dependence can then be added to the solution once the field distributions are well known. The solution this problem was obtained by Jackson [17]:

$$
\begin{gathered}
\Phi_{\text {in }}=-\frac{3 \varepsilon_{N P}}{\varepsilon_{N P}+2 \varepsilon_{m}} E_{0} r \cos \theta \\
\Phi_{\text {out }}=-E_{0} r \cos \theta+\frac{\varepsilon_{N P}-\varepsilon_{m}}{\varepsilon_{N P}+2 \varepsilon_{m}} E_{0}\left(\frac{d}{2}\right)^{3} \frac{\cos \theta}{r^{2}}
\end{gathered}
$$

$E_{0}$ is the amplitude of the electric field, $\varepsilon_{N P}$ and $\varepsilon_{m}$ are the dielectric permittivity of the NP and of the surrounding medium respectively, both are function the excitation frequency $\omega, r$ is the module of the position vector. Here $\Phi_{\text {out }}$ describes the superposition of the applied field and that of a dipole located at the NP center, i.e., an applied field induces dipole moment inside the sphere proportional to $\left|E_{0}\right|$. Additionally, we can arrive at

$$
\alpha=4 \pi\left(\frac{d}{2}\right)^{3} \frac{\varepsilon_{N P}-\varepsilon_{m}}{\varepsilon_{N P}+2 \varepsilon_{m}}
$$

Here $\alpha$ is the (complex) polarizability of the NP in the electrostatic approximation. Under the condition that $\left|\varepsilon_{N P}+2 \varepsilon_{m}\right|$ is a minimum, the polarizability shows a resonant enhancement. Thus, we can also write the Frohlich condition $\operatorname{Re}\left[\varepsilon(\omega)_{N P}\right]=-2 \varepsilon_{m}$ that is the associated mode the dipole surface plasmon of the NP (in an oscillating field). Nevertheless, the distribution of the electric field $E_{\text {in }}$ inside and $E_{\text {out }}$ outside the sphere can be written as:

$$
\begin{gathered}
E_{\text {in }}=\frac{3 \varepsilon_{m}}{\varepsilon_{N P}+2 \varepsilon_{m}} E_{0} \\
E_{\text {out }}=E_{0}+\frac{3 \mathrm{n}(\mathrm{n} \cdot \mathrm{p})-\mathrm{p}}{4 \pi \varepsilon_{0} \varepsilon_{m}}\left(\frac{1}{r}\right)^{3}
\end{gathered}
$$

Here, $\mathrm{p}=\varepsilon_{0} \varepsilon_{\mathrm{m}} \alpha E_{0}$ is the dipole moment. Therefore, a resonance in $\alpha$ implies a resonant improvement of both the internal and dipolar fields, resulting in prominent applications of NPs in optical devices. Now consider a plane wave incident with $E(r, t)=E_{0} e^{-i \omega t}$, this induce on NP an oscillating dipole moment $\mathrm{p}(t)=\varepsilon_{0} \varepsilon_{\mathrm{m}} \alpha E_{0} e^{-i \omega t}$, i.e., we have a scattering of the plane wave by the NP. In this sense, the electromagnetic fields associated with an electric dipole in the near, intermediate and radiation zones are [17]:

$$
H=\frac{c k^{2}}{4 \pi}(\mathrm{n} \times \mathrm{p}) \frac{e^{i k r}}{r}\left(1-\frac{1}{i k r}\right)
$$




$$
E=\frac{1}{4 \pi \varepsilon_{0} \varepsilon_{m}}\left\{k^{2}(\mathrm{n} \times \mathrm{p}) \times \mathrm{n} \frac{e^{i k r}}{r}+(3 \mathrm{n}(\mathrm{n} \cdot \mathrm{p})-\mathrm{p})\left(\frac{1}{r^{3}}-\frac{i k}{r^{2}}\right) e^{i k r}\right\}
$$

With $k=2 \pi / \lambda$ and $\mathrm{n}$ is the unit vector in the direction of the point $\mathrm{P}$ of interest. For $k r<<1$ (near zone) we have the electrostatic result of (5). The magnetic field present has the form $H=\frac{i \omega}{4 \pi} \frac{(\mathrm{n} \times \mathrm{p})}{r^{2}}$. Hence, in the near field the fields are predominantly electric and for static field $(k r \rightarrow 0)$, the magnetic field vanishes. For $k r>>1$, the dipole fields have spherical-wave form: $H=\frac{c k^{2}}{4 \pi} \frac{(\mathrm{n} \times \mathrm{p}) e^{i k r}}{r}$ and $E=\sqrt{\frac{\mu_{0}}{\varepsilon_{0} \varepsilon_{m}}} H \times \mathrm{n}$.

From the viewpoint of optics, it is much interesting to note that another consequence of the enhanced polarization $\alpha$ in which a NP scatters and absorbs light [18]. For a sphere of volume $V$ and dielectric function $\varepsilon_{N P}=\varepsilon_{1}+i \varepsilon_{2}$ in the quasi-static limit, the extinction cross section $C_{e x t}=C_{a b s}+C_{s c a}$ is:

$$
C_{e x t}=9 \frac{\omega}{c} \varepsilon_{m}^{3 / 2} \frac{\varepsilon_{2}}{\left(\varepsilon_{1}+2 \varepsilon_{m}\right)^{2}+\varepsilon_{2}^{2}}
$$

\subsection{Mie theory}

For particles with larger dimensions, where the quasi-static approximation is not justified due to significant phase-changes of the driving field over the particle volume, a rigorous electrodynamics approach is required. This way, Gustav Mie solved Maxwell's equations for the case of an incoming plane interacting with a spherical particle [19]. In essence, the electromagnetic fields are expanded in multipole contributions and the expansion coefficients are found by applying the correct boundary conditions for electromagnetic fields at the interface between the metallic NP and its surrounding.

The extinction cross section of a spherical NP is given by the following expression:

$$
\sigma_{e x t}=\frac{\lambda^{2}}{2 \pi} \sum_{n=0}^{\infty}(2 n+1) \operatorname{Re}\left\{a_{n}+b_{n}\right\}
$$

Here the parameters $a_{n}$ and $b_{n}$ are defined as:

$$
\begin{aligned}
& a_{n}=\frac{\Psi_{n}(\beta) \Psi_{n}^{\prime}(m \beta)-m \Psi_{n}(m \beta) \Psi_{n}^{\prime}(\beta)}{\xi_{n}(\beta) \Psi^{\prime}{ }_{n}(m \beta)-m \Psi_{n}(m \beta) \xi_{n}^{\prime}(\beta)} \\
& b_{n}=\frac{m \Psi_{n}(\beta) \Psi_{n}^{\prime}(m \beta)-\Psi_{n}(m \beta) \Psi_{n}^{\prime}(\beta)}{m \xi_{n}(\beta) \Psi^{\prime}{ }_{n}(m \beta)-\Psi_{n}(m \beta) \xi_{n}^{\prime}(\beta)}
\end{aligned}
$$


The size parameter $\beta$ is defined as $\beta=\frac{\pi d m_{0}}{\lambda_{0}}$, where $\lambda_{0}$ is the incident wavelength with respect to vacuum, and $m_{0}$ represents the refractive index of the surrounding medium. The Ricatti-Bessel functions $\Psi$ and $\xi$ are defined in terms of the half-integer-order Bessel function of the first kind $\left(J_{n+1 / 2}(z)\right), \Psi_{n}(x)=\left(\frac{r x}{2}\right)^{0.5} J_{n+1 / 2}(x)$ and $\xi_{n}(x)=\left(\frac{r x}{2}\right)^{0.5} H_{n+1 / 2}(x)$, $H_{n+1 / 2}(x)$ is the half-integer-order Hankel function of the second kind.

We will focus our attention in silver and gold, since the localized plasmon resonance condition mentioned above is satisfied at visible light frequencies. Additional advantages of these metal NPs include simple preparation methods for a wide range of sizes and shapes and easy surface conjugation to a variety of ligands.

We now evaluate the extinction cross section using the Mie theory from equation (9), Figure 2, we take dielectric constants for silver and gold from Palik [20], and the medium dielectric constant is assumed to be 1.0 (i.e., a particle in a vacuum) and 2.0 for NPs with sizes different.

We can see from Figure 2 the dependence of resonance frequency with size of NPs (silver and gold) and the refraction index (surrounding medium). K. Lance Kelly et al. (2003), show that for spherical NPs clear differences between the quasistatic and the Mie theory results. However, the important features are retained, e.g. the frequency resonance. Although Mie theory is not a very expensive calculation, the quasistatic expressions are convenient to use when only qualitative information is needed [21].

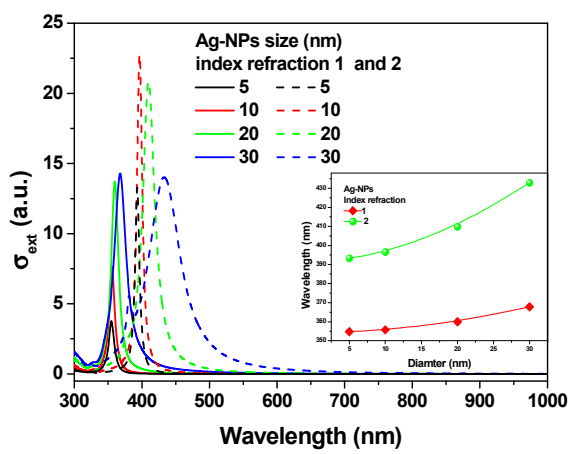

(a)

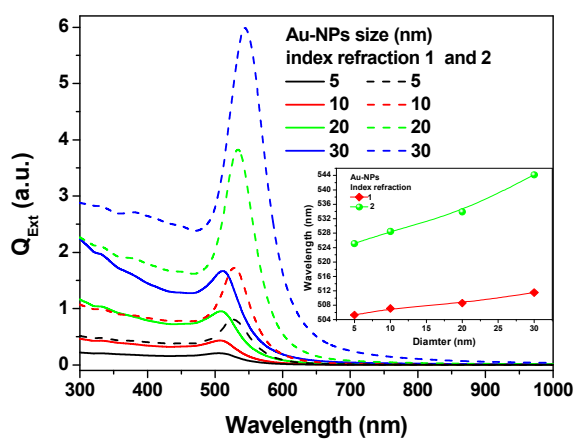

(b)

Figure 2. Extinction cross section from Mie theory for (a) silver and (b) gold NPs. Both in function of size with refraction index 1 (line curves) and 2 (dot lines). Inset figure shows a red-shift with the increment of size NPs and refraction index.

Now, Figure 3 shows clearly the dependence of resonance frequency embedded in a glass (with refraction index of: 1.99 for $400 \mathrm{~nm}, 1.88$ for $630 \mathrm{~nm}, 1.75$ for $900 \mathrm{~nm}$ and 1.70 for 1000 $\mathrm{nm}$ ) and in a bulk with refraction index 2, in both cases with $20 \mathrm{~nm}$ size and a size 
distribution of $20 \%$ STD, this simulation was extracted from program MiePlot v4.2.11 of Philip Laven. Also, it is possible to observe an increment of bandwidth and the intensity of peak for NPs embedded in the glass when compared with the material bulk. Therefore, LSPR results in enhanced local electromagnetic fields near the surface of the NP (Novotny \& Hecht, 2006) [22], see Figure 1.

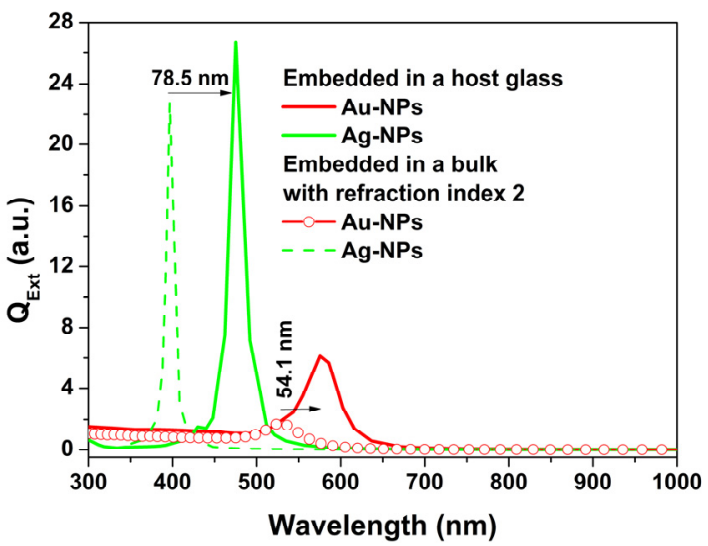

Figure 3. Extinction cross section from Mie theory via the program MiePlot v4.2.11 of Philip Laven, for silver and gold NPs with a size distribution of $20 \%$ STD of $20 \mathrm{~nm}$ size embedded in a glass with refraction index in function of the wavelength and a bulk with refraction index 2 constant. In both cases, we observed a red-shift (see arrow). Inset figure shows the size distribution of NPs for 50 NPs in both cases.

Hideki Nabika and Shigehito Deki [23], show an experimental evidence of this dependence above mentioned for silver NPs of different sizes were synthesized by reducing $\mathrm{AgNO}_{3}$ with $\mathrm{N}, \mathrm{N}$-dimethylformamide (DMF), Poly(vinylpyrrolidone) (PVP) aqueous solution (10 mL, 10 wt \%) was added to DMF ( $80 \mathrm{~mL})$, followed by an addition of $\mathrm{AgNO}_{3}$ aqueous solution (10 $\mathrm{mL}$ ). They obtained three sets of spherical silver NPs with a particle diameter varying from 9.7 to $27.1 \mathrm{~nm}$ and rod-shaped silver NPs with an aspect ratio of 1.79 , its results are show Figure 4 .

Thereby, the LSPR properties are sensitive to its environment in the order of its shape and size [24], the dielectric function of the glass host containing the NPs, changes the LSPR bandwidth and resonance frequency, see Figure 2-4.

We can be written as the sum of the bound and quasi-free (conduction) electron contributions [25]: $\varepsilon(\omega)=\varepsilon^{i b}(\omega)-\omega_{p}^{2} / \omega[\omega+i \gamma], \gamma$ is the damping the resonance, $\omega$ the excitation frequency, $\omega_{p}$ the plasma frequency. The bound electron contribution $\varepsilon^{i b}$ remains unchanged [26]. A similar expression can be used for the contribution of conduction electrons [27]: $\gamma=1 / \tau^{N P}=1 / \tau_{0}+2 g_{s} V_{F} / d$. Where the first term, $1 / \tau_{0}$ is associated to bulklike electron scattering process in the particle and the second term is a consequence of quasi-electron-free interaction with the surface and, for a sphere, $V_{F}$ is the Fermi velocity, and $g_{s}$ is the surface factor [25]. Nevertheless, the LSPR dependence on the matrix refractive 
index $\left(n_{\lambda}\right)$ also can be calculated by [12]: $\omega_{p}=\sqrt{4 \pi n^{\prime} e^{2} / \varepsilon_{0} \varepsilon_{d}(\omega) m^{*}}$, where $n^{\prime}$ is the electrons density, $e$ the electron charge, $\left(\varepsilon_{d}(\omega)=n_{\lambda}^{2}\right)$ the dielectric permittivity, and $m^{*}$ the electron mass.

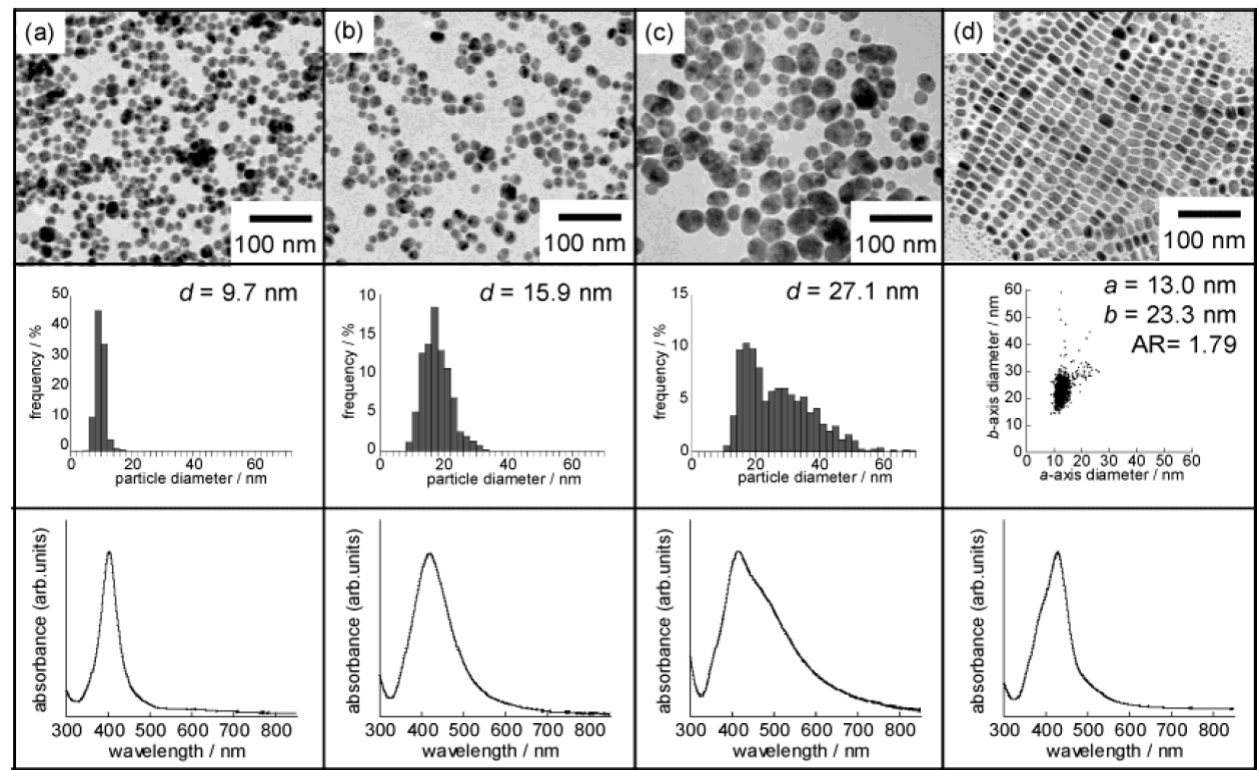

Figure 4. TEM micrographs, size distributions and optical absorption spectra of spherical silver NPs. Hideki Nabika and Shigehito Deki [23]. (a) $90{ }^{\circ} \mathrm{C}, \mathrm{AgNO}_{3}=0.125 \mathrm{M}$, (b) $90{ }^{\circ} \mathrm{C}, \mathrm{AgNO}_{3}=0.250 \mathrm{M}$, and (c) $110^{\circ} \mathrm{C}, \mathrm{AgNO}_{3}=0.250 \mathrm{M}$ and $(\mathrm{d})$ of the rod-shaped silver NPs.

On the other hand, in the Gans theory, the LSPR is only a function of the aspect ratio and refractive index. Thus, in certain conditions, a linear relationship between them can be resulted [28]. Nevertheless, numerical results suggest that, even when the aspect ratio is fixed and the retardation effect is weak, the position of longitudinal resonance can still depend strongly with the aspect ratio $[29,30]$. Using the model of Cheng - ping Huang et al [31], we can write:

$$
\lambda_{p}=\pi n \sqrt{10 \kappa\left(2 \delta^{2}+r^{2} \ln [\kappa]\right)}
$$

Where $\kappa=l / 2 r$ is the aspect ratio of the NPs (with the inner radius $r$ and in a cylindrical region with the length $l$ ), and $\delta$ is the skin depth. Cheng - ping Huang considered the problem as using an LC circuit model without solving the Laplace or Maxwell equations, and show that its results overcomes the deficiency of Gans theory and provides a new insight into the phenomenon. This way, we can observe resonance wavelength change with the aspect ratio of NP, Figure 5. This means a breakdown of the linear behavior presenting oscillations electrons originating from the amorphous geometry of the NPs associated with the inertia of electrons, see inset Figure 2 as well. 


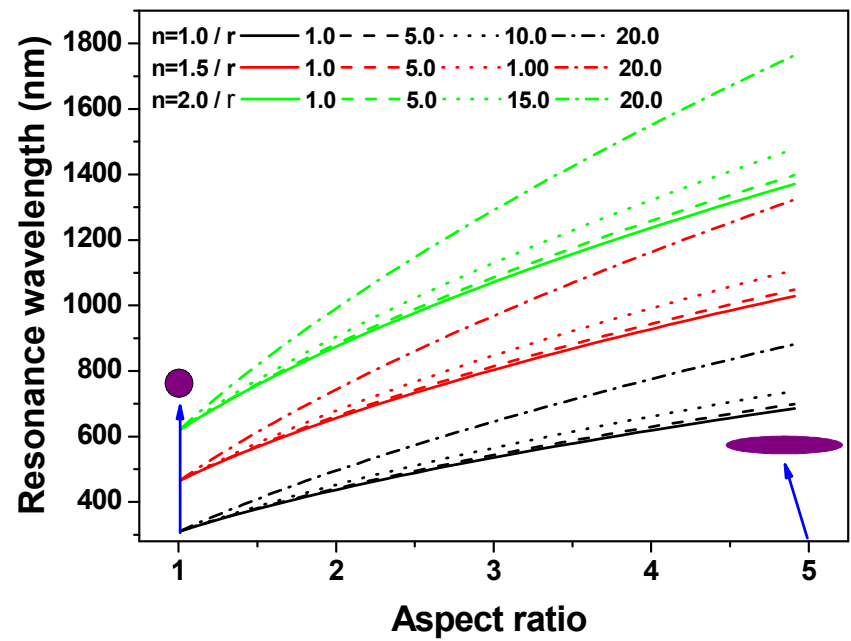

Figure 5. Resonance wavelength with aspect ratio from Cheng - ping Huang prediction for radius different and embedded in a bulk with refraction index 1.0, 1.5 and 2.0.

Figure 6 illustrates this further, via experiments of X. Lu et al. [15] and Mock et al. [32]. Panel (a) the nanobar Ag NPs and the corresponding dark-field light scattering spectra, in broad agreement with the simulated results shown in [15]. Panel (b) shows the dipolar plasmon lineshapes of colloidal silver NPs of different shapes [32].

\subsection{Coupling between metallic nanoparticles}

We can say that the localized plasmon resonance frequency of a single metallic NP can be shifted through of alterations in shape, size and surrounding medium from the Frohlich condition, section 2.1. Nevertheless, in a NP ensembles we can obtain additional shifts due to electromagnetic interactions between the localized modes, see Figure 3 and 4 (c). These interactions are basically of a dipolar nature (when $d<<\lambda$ ). So, the NP ensemble can be treated as an ensemble of interacting dipoles (in a first approximation). Those NPs can be embedded into a host matrix ordered or random, in one-, two- or three-dimensional arrays with interparticle spacing $D$. Electromagnetic coupling of those arrays shows interesting localization effects for closely spaced particles such as, enhancement process due to field localization in NP junctions. Assuming a dipolar approximation the NPs can be treated as point dipoles. In this sense, two regimes have to be distinguished (i) For $D<<\lambda$, near-field interactions with a distance dependence of $D^{-3}$ dominate, equation (5), and the NP array is described as an array of point dipoles coupling via their near-field, see section 2.1 and Figure 7. These arrays can serve as hot-spots for field enhancement, e.g. in a context of surface-enhanced Raman scattering (SERS). (ii) For larger NP separations, far-field dipolar coupling with a distance dependence of $D^{-1}$ dominates, see section 2.1.

Therefore, the maximum field enhancement is determined by the shortest distance between two equipotential particles. 


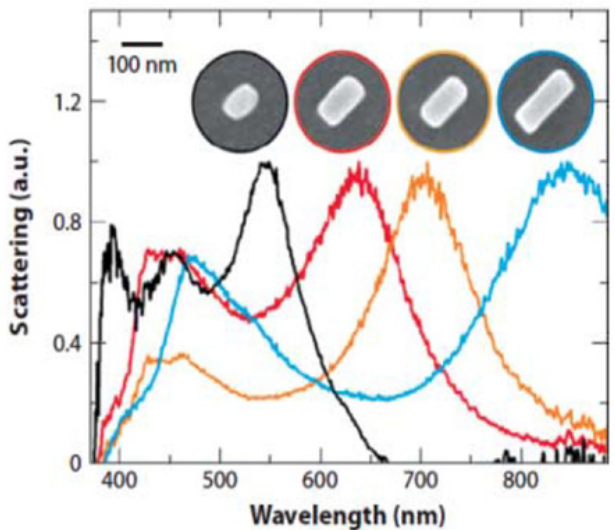

(a)

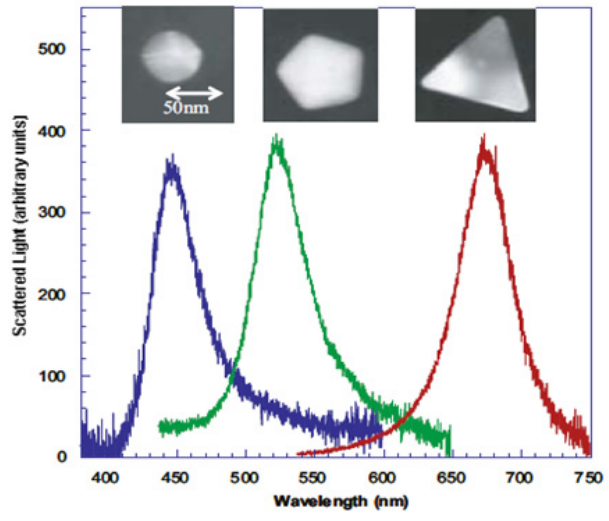

(b)

Figure 6. Panel (a) scattering spectra. In the insets are SEM images of the individual Ag nanobars. X. Lu et al. [15]. For simple, highly symmetric shapes (sphere, cube and triangular plate), the spectra are dominated by a single peak, but with the peak position sensitive to the shape, and ranging from 400 to up $800 \mathrm{~nm}$. Panel (b) Scattering spectra of single silver nanoparticles of different shapes obtained in dark-field configuration. Mock et al. [32].

Figure 7 shows the near field coupling between NPs. Here, the restoring force acting on the oscillating electrons of each NP in the chain is either increased or decreased by the charge distribution of neighboring particles. Depending on the polarization direction of the exciting light, this leads to a blue-shift of the plasmon resonance for the excitation of transverse modes, Figure 7 (a), and a red-shift for longitudinal modes, Figure 7 (b).

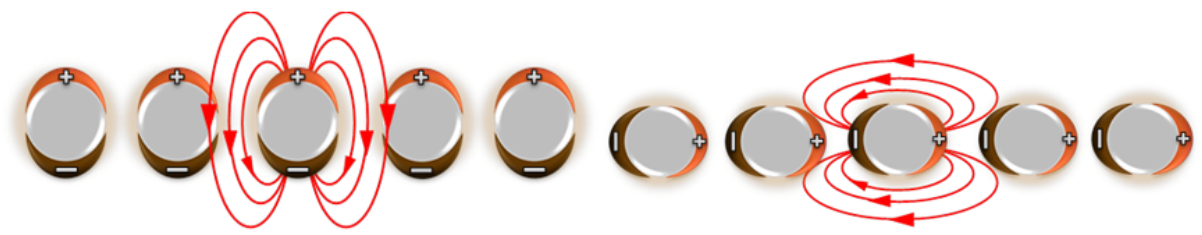

Figure 7. Schematic of near-field coupling between metallic NPs for two different polarizations.

Today, there are several theoretical methodologies available to describe the interaction among the metallic NPs with electromagnetic radiation $[33,34,35,36,37,38,39,40]$.

\section{Rare-earths}

Rare earths ions in solids are either divalent or trivalent. Their electronic configuration is

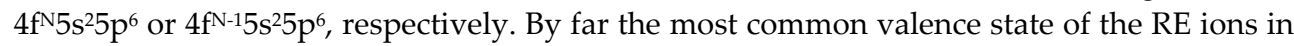
solids is the trivalent one. Those ions have a long history in optical and magnetic applications. We have special interest in the devices luminescent using crystal, powders, and glasses. On the other hand, divalent RE ions have also been used in laser devices, but only in relatively exotic ones for cryogenic operation [41,42]. The most frequently used laser- 
active RE ions and host media together with typical emission wavelength ranges are shown in the table 1 :

\begin{tabular}{|c|c|c|}
\hline Ion & Common host media & $\begin{array}{l}\text { Important emission } \\
\text { wavelengths }(\mu \mathrm{m})\end{array}$ \\
\hline Neodymium $\left(\mathrm{Nd}^{3+}\right)$ & YAG, $\mathrm{YVO}_{4}, \mathrm{YLF}$, silica & $1.03-1.1,0.9-0.95,1.32-1.35$ \\
\hline Ytterbium $\left(\mathrm{Yb}^{3+}\right)$ & YAG, tungstates, silica & $1.0-1.1$ \\
\hline Erbium $\left(\mathrm{Er}^{3+}\right)$ & $\begin{array}{l}\text { YAG, silica, tellurite, chacogenetos } \\
\text { glasses }\end{array}$ & $1.5-1.6,2.7,0.55$ \\
\hline Thulium $\left(\mathrm{Tm}^{3+}\right)$ & YAG, silica, fluoride glasses & $1.7-2.1,1.45-1.53,0.48,0.8$ \\
\hline Holmium $\left(\mathrm{Ho}^{3+}\right)$ & YAG, YLF, silica & $2.1,2.8-2.9$ \\
\hline $\begin{array}{l}\text { Praseodymium } \\
\left(\operatorname{Pr}^{3+}\right)\end{array}$ & silica, fluoride glasses & $1.3,0.635,0.6,0.52,0.49$ \\
\hline Cerium $\left(\mathrm{Ce}^{3+}\right)$ & $\begin{array}{l}\text { YLF, LiCAF, LiLuF, LiSAF, and similar } \\
\text { fluorides }\end{array}$ & $0.28-0.33$ \\
\hline
\end{tabular}

Table 1. Common laser-active rare earth ions and host media and important emission wavelengths.

The $4 \mathrm{f}$ electrons are not the outermost ones. They are shielded from external fields by two electronic shells with larger radial extension $\left(5 \mathrm{~s}^{2} 5 \mathrm{p}^{6}\right)$, which explains the atomic nature of their spectra absorption/emission, Figure 8 . Thus the 4 f electrons are only weakly perturbed by the charges of the surrounding atoms. Important characteristics that distinguish them from other optically active ions are: (i) the wavelengths of the emission and absorption transitions are relatively insensitive to host material, (ii) the intensities of these transitions are weak, (iii) the lifetimes of metastable states are long and, (iv) the quantum efficiencies tend to be high. This is why the RE ions are such a useful probe in a solid; the crystal environment constitutes only a small perturbation on the atomic energy levels, and many of the solid state, and hence spectroscopic, properties can be understood from a consideration of the free ions. In turn, the wavefunctions of the free ions constitute a good zero order approximation for a description of solid-state properties, Figure 8.

The solutions to this problem can then befactored into a product of a radial and angular function. Whereas the radial function depends on the details of the potential the spherical symmetry ensures that the angular component is identicalwith that of a hydrogen atom and can be expressed as spherical harmonics. Except for $\mathrm{Ce}^{3+}$ and $\mathrm{Yb}^{3+}$, which have only one electron, the solutions of the central-field problem are products of one-electron states that are antisymmetric under the interchange of a pair of electrons, as required by the Pauli exclusion principle.

In describing the state of a multielectron atom, the orbital angular momenta and the spin angular momenta are added separately. The sum of the orbital angular momenta is designated by the letter $L$, and the total spin is characterized by $S$. The total angular momentum $J$ of the atom may then be obtained by vector addition of $L$ and $S$. The collection of energy states with common values of $J, L$, and $S$ is called a term. Here, the Russell- 
Saunders notation for the energy levels (terms) is used, ${ }^{2 S+1} L$, this takes into account the spin-spin coupling, orbit-orbit coupling and spin-orbit coupling. The energy levels of a free RE ion are usually interpreted by considering only interactions between the $4 \mathrm{f}$ electrons themselves. Since all the other electronic shells are spherically symmetric, Figure 8, their effect on all the terms of a configuration is the same in first order, and therefore do not contribute significantly to the relative positions of the $4 \mathrm{f}$ energy levels, we can write:

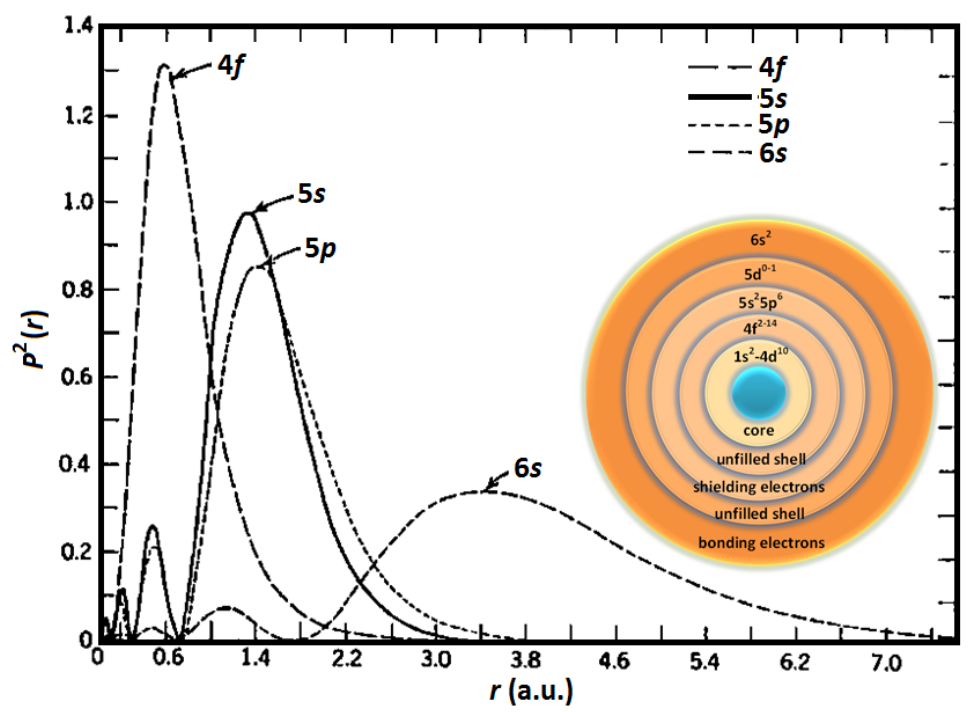

Figure 8. Square of the radial wavefunctions for the $4 \mathrm{f}, 5 \mathrm{~s}, 5 \mathrm{p}$ and $6 \mathrm{~s}$ energy levels from Hartree-Fock. It was a calculation for $\mathrm{Gd}^{+}$by Freeman e Watson (1962) [46]. Picture inset schematically shows that the $4 \mathrm{f}$ orbital is within the $6 \mathrm{~s}, 5 \mathrm{p}$ and $5 \mathrm{~s}$ levels.

$$
H=-\frac{\hbar^{2}}{2 m} \sum_{i=1}^{N} \Delta_{i}-\sum_{i=1}^{N} \frac{Z^{*} e^{2}}{r_{i}}+\underbrace{\sum_{i<j}^{N} \frac{e^{2}}{r_{i j}}}_{H_{C}}+\underbrace{\sum_{i=1}^{N} \zeta\left(r_{i}\right) s_{i} \cdot l_{i}}_{H_{S O}}+V_{E F}
$$

Where $N=1 \ldots 14$ is the number of the $4 \mathrm{f}$ electrons, $Z^{*} e$ the screened charge of the nucleus because we have neglected the closed electronic shell, $V_{E F}$ treats the interaction of the ion with the electromagnetic field, and $\zeta\left(r_{i}\right)$ the spin-orbit coupling function [43],

$$
\zeta\left(r_{i}\right)=\frac{\hbar^{2}}{2 m^{2} c^{2} r_{i}} \frac{d U\left(r_{i}\right)}{d r_{i}}
$$

Where $U\left(r_{i}\right)$ is the potential in which the electron $i$ is moving. The first two terms of the Hamiltonian (equation (13)) are spherically symmetric and therefore do not remove any of the degeneracies within the configuration of the $4 \mathrm{f}$ electrons, therefore we can neglect. The next two terms, which represent the mutual Coulomb interaction of the 4 f electrons $\left(H_{c}\right)$ and their spin-orbit interaction $(\mathrm{Hso})$ are responsible for the energy level structure of the $4 \mathrm{f}$ 
electrons. Details of the matrix element calculations of $H_{c}$ and $H_{s o}$ can be found in ref. $[44,45]$.

Figure 8 shows the radial distribution functions $4 \mathrm{f}, 5 \mathrm{~s}, 5 \mathrm{p}$, and $6 \mathrm{~s}$ electrons for $\mathrm{Gd}^{+}$as obtained from Harteww-Fock calculation by Freeman and Watson [46]. We can see that the 4 f electrons are inner electrons with relatively small $\left\langle r^{n}\right\rangle$ values. As we shall see, the crystal field interaction will be small in REs. It is also evident that the $4 \mathrm{f}$ wavefunctions do not extend very far beyond the $5 \mathrm{~s}$ and $5 \mathrm{p}$ shells. Thus, all quantities of a solid depend on an overlap of the $4 \mathrm{f}$ wavefunctions with those of a neighboring ion have to be small in RE compounds.

\subsection{Trivalent ions in a static crystal field}

The $4 \mathrm{f}$ shell of the RE ions are an unfilled shell and therefore have a spherical charge distribution. If the ion is introduced into a crystal, the ion experiences an inhomogeneous electrostatic field, the so-called crystal field, which is produced by the charge distribution into the crystal. This crystal field distorts the closed shells of the RE ion. Producing an effect on the energy level $4 \mathrm{f}$, i.e., removes to a certain degree the degeneracy of the free ion $4 \mathrm{f}$ levels, thus producing a major modification of the energy levels (but this depends on the crystal symmetry). In the luminescence spectrum are observed additional transitions, which originate from the excited crystal field levels of the ground term is evident, and the spectra look more complicated. On the other hand, these spectrums can be used to determine the crystal field energies of the ground term.

We shall now try to understand the behavior of these additional transitions. To do so we draw on our treatment of the Coulomb interaction of the $4 \mathrm{f}$ electrons. The crystal field interaction comes by the interaction of the $4 \mathrm{f}$ electrons with all the charges of the crystal with all charges of the crystal, except for trivial factors, it is therefore given by one over the radius vector between the $4 \mathrm{f}$ electrons and the crystal charges. This function is given, apart from radial factors, of two spherical harmonics $Y_{k q}$, one containing the coordinates of the $4 \mathrm{f}$ electrons, the other containing coordinates of the crystal charge. The latter has to be integrated over the whole crystal, whereupon it gives the strength of the crystal potential at the site of the RE ion. Finally, this shows the existence of the splitting telling us that the development of the crystal potential into spherical harmonics contains a second order term.

\subsubsection{Crystal field splitting}

Crystal field splitting has two aspects: (i) symmetry, namely, the number of the levels into which an ion is free the $J$ term are split in a crystal field of a given symmetry; (ii) the actual size of the crystal field splitting.

This way, the point charge model can be used for illustrative purposes but not for a quantitative description of the crystal field interaction. A realistic description of this interaction must take into account that the crystal field is built up of the spatially extended charge clouds of the individual ions. Thus, these charge clouds can penetrate each other and thereby interact. Hence, we can considered the following elements: 
1. The ions are considered static in the crystal, i.e., we can neglected the lattice vibrations and their effect on the energy levels.

2. We regard the $4 \mathrm{f}$ electrons of one RE ion, as representative of those of all the RE ions in the crystal and, thus the interaction of $4 \mathrm{f}$ electrons of adjacent ions is neglected.

3. The crystal consists of the extended charge distributions. This produces an overlap of the charge distributions of the neighboring ions and the $4 \mathrm{f}$ electrons. Aditionally, a charge transfer between $4 \mathrm{f}$ electrons and the electrons of the ligands can take place. Both contribute to the crystal field interaction.

4. The $4 \mathrm{f}$ electrons of one ion are considered to be independent of each other, that is correlation effects play no significant role.

Therewith, we can calculate the crystal potential $\Phi\left(r_{i}, \varphi_{i}, \theta_{i}\right)$ at the site of the $4 \mathrm{f}$ electrons and the potential energy of the $4 \mathrm{f}$ electrons in this potential. If the crystal has charge density $\rho(R)$ and the $4 \mathrm{f}$ electrons have radius $r_{i}$, we have:

$$
V=-\sum_{i} \int \frac{e_{i} \rho(R)}{\left|R-r_{i}\right|} d \tau=-\sum_{k, i} e_{i} \int \rho(R) P_{k}\left(\cos \left[R, r_{i}\right]\right) \frac{r_{<}^{k}}{r_{>}^{k+1}} d \tau
$$

Where $r<$ and $r>$ are, respectively, the smaller and larger value of $r$ and $r_{i}$. Here $P_{k}(\cos [x])$ are the Legendre polynomials. Also, the equation (15) can be re-write as:

$$
V=-\sum_{k, q, i} B_{k, q} C_{k, q}\left(\theta_{i}, \varphi_{i}\right)
$$

Where the crystal field parameter $B k, q$ ( $k \leq 6$ for $f$ electrons) and the summation $i$ is carried out over all the $4 f$ electrons of the ions. $B k, q$ have the form:

$$
B_{k, q}=-e \int(-1)^{q} \rho(R) C_{k-q}(\theta, \varphi) \frac{r_{<}^{k}}{r_{>}^{k+1}} d \tau
$$

Here $C_{k, q}$ is called a tensor operator and is defined as: $C_{k, q}=\left(\frac{4 \pi}{2 k+1}\right)^{0.5} Y_{k, q}$

In early treatments of the crystal fiel interaction it was assumed that in RE compounds the point charge contribution would be the dominant part of the crystal field interaction. Then, in equation (16) the integral over the lattice can be replaced by a sum over all lattice points and $r_{<}^{k}$ can be replaced by $r_{i}^{k}$. The latter replacement can be performed as long as the charge distribution of the crystal does not enter that of the $4 \mathrm{f}$ electrons (as long as $r_{i}<R$ ), which implies that the potential acting on the $4 \mathrm{f}$ electrons obeys the Laplace equation $(\Delta \Phi(r, \varphi, \theta)=0)$ at the position of the 4 f electrons.

Figure 9 further illustrates the effect of spin-orbit and crystal field interactions on the energy levels of the $\mathrm{Er}^{3+}$ ion. This figure were extracted from A.J. Kenyon [47]. A further splitting of the energy levels comes about when the ion is placed in a silica host. The $4 \mathrm{f}^{\mathrm{N}}$ configuration is composed for a number of states where the quantum numbers $(L, S, J$, and another arbitrary 
one) define the terms of the configuration, all of which are degenerate in the central-field approximation, as illustrated in Figure 9. Next in the hierarchy is spin-orbit, the strongest of the magnetic interactions. Spin-orbit lifts the degeneracy in total angular momentum and splits the LS terms into J levels, Figure 9.

We can see that the environment provided by silica hosts destroys the spherically symmetric environment that $\mathrm{Er}^{3+}$ ions enjoy in the vapor phase. Thus the degeneracy of the $4 \mathrm{f}$ atomic states will be lifted to some degree. This splitting is also referred to as stark splitting, and the resulting states are called Stark components (of the parent manifold). The even- $k$ terms in the expansion split the free ion $J$ multiplets into Stark components generally separated by $10-100 \mathrm{~cm}^{-1}$ (see Figure 9). The ion-lattice interaction can mix multiplets with different $J$ values $(J$ mixing), although it usually remains a good quantum number. The odd- $k$ terms admix higher lying states of opposite parity [e.g., $4 \mathrm{f}^{\mathrm{N}} 15 \mathrm{~d}^{1}$ ] into the $4 \mathrm{f}^{\mathrm{N}}$ configuration. This admixture does not affect the positions of the energy levels, but it has a very important effect on the strengths of the optical transitions between levels.

\subsection{Optical properties}

In recent years most of the interest in luminescent RE ions has concentrated on the trivalent erbium $\left(\mathrm{Er}^{3+}\right)$, and in particular its emission band around $1550 \mathrm{~nm}$. This emission is within the telecommunication windows, and fortuitously coincides with the $1550 \mathrm{~nm}$ intra- $4 \mathrm{f}$ ${ }^{4} \mathrm{I}_{13 / 2} \rightarrow{ }^{4} \mathrm{I}_{15 / 2}$ transition of the $\mathrm{Er}^{3+}$ ion.

For this reason exists today a great interest in utilizing erbium-doped materials for gain elements and sources in telecommunications systems $[48,49,50]$. The development of the erbium-doped fiber amplifier (EDFA) in the late 1980s [51,52] exploited the ${ }^{4} \mathrm{I}_{13 / 2} \rightarrow{ }^{4} \mathrm{I}_{15 / 2}$ transition and allowed the transmission and amplification of signals in the 1530-1560 nm region without the necessity for expensive optical to electrical conversion [53]. Hence, it is originated a driving force for research in RE doped fibers and integrated optics waveguides has been their use for amplifying weak signals in optical communications systems at 1300 and $1600 \mathrm{~nm}$. This may be achieved by simply splicing a section of RE-doped fiber into the transmission one and injecting pump light through a fiber coupler. The signal generated within the RE emission band stimulates emission of radiation at the same frequency, amplifying the optical communication signal with high gain, high efficiency and low noise, which is highly advantageous for optical communications [54]. There are five main RE candidates for use as dopants in fiber or waveguide amplifiers for optical communications systems: $\mathrm{Er}^{3+}, \mathrm{Tm}^{3+}, \mathrm{Nd}^{3+}, \mathrm{Pr}^{3+}$ and $\mathrm{Dy}^{3+}$. $\mathrm{The}^{\mathrm{Er}^{3+}}$ and $\mathrm{Tm}^{3+}$ ions are the choice for the 1400$1600 \mathrm{~nm}$ window centered at $1550 \mathrm{~nm}$, based on the ${ }^{4} \mathrm{I}_{13 / 2} \rightarrow{ }^{4} \mathrm{I}_{15 / 2}$ transition of $\mathrm{Er}^{3+}$ ion and the ${ }^{3} \mathrm{H}_{4} \rightarrow{ }^{3} \mathrm{~F}_{4}$ transition of $\mathrm{Tm}^{3+}$ ion. The ${ }^{4} \mathrm{~F}_{3 / 2} \rightarrow{ }^{4} \mathrm{I}_{13 / 2}$ emission of $\mathrm{Nd}^{3+}$ ion, the ${ }^{1} \mathrm{G}_{4} \rightarrow{ }^{3} \mathrm{H}_{5}$ transition of $\mathrm{Pr}^{3+}$ ion and the ${ }^{6} \mathrm{~F}_{11 / 2}\left({ }^{6} \mathrm{H}_{9 / 2}\right) \rightarrow{ }^{6} \mathrm{H}_{15 / 2}$ transition of $\mathrm{Dy}^{3+}$ ion are all potentially useful for the $1300 \mathrm{~nm}$ telecommunication window.

Further, the $3+$ ions all exhibit intense narrow-band intra- 4 f luminescence in a wide variety of hosts, and the shielding provided by the $5 \mathrm{~s}^{2}$ and $5 \mathrm{p}^{6}$ electrons (see Figure 8 ), which means that rare-earth radiative transitions in solid hosts resemble those of the free ions and 
electron-phonon coupling is weak. Although some of the divalent species also exhibit luminescence (e.g., samarium and europium), it is the trivalent ions that are of most interest.

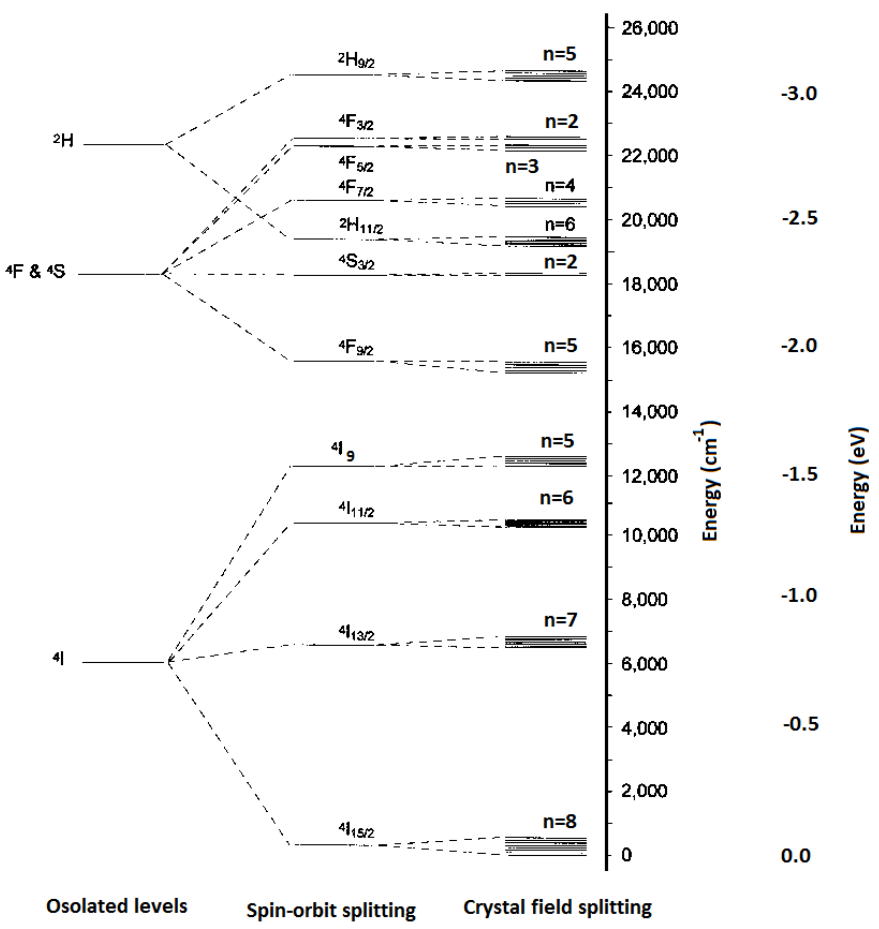

Figure 9. The effect of spin-orbit and crystal field splitting on the energy levels of the $\mathrm{Er}^{3+}$ ion in silica matrix. The energy diagram shows the hierarchy of splitting resulting from electron-electron and electron-host interactions. Figure extracted from A.J. Kenyon [47].

As mentioned previously, the intra- $4 \mathrm{f}$ transitions are parity forbidden and are made partially allowed by crystal field interactions mixing opposite parity wavefunctions, resulting in luminescence lifetimes long (often in the millisecond range), and linewidths narrow. The selection of an appropriate ion with intense and narrow-band emission can be obtained across much of the visible region and into the near-infrared. Figure 10 shows energy level diagrams for the isolated 3+ ions of each of the 13 lanthanides with partially filled $4 \mathrm{f}$ orbitals.

Almost all the RE ions their emission is due to optical transitions within the f-manifold (e.g. $\mathrm{Tb}^{3+}\left(4 \mathrm{f}^{8}\right), \mathrm{Gd}^{3+}\left(4 \mathrm{f}^{7}\right)$ and $\mathrm{Eu}^{3+}\left(4 \mathrm{f}^{7}\right)$. The $4 \mathrm{f}$-electrons are well shielded from the chemical environment and therefore have almost retained their atomic character. Nevertheless, for a number of RE ions, also broad emission bands are known. Prominent examples are $\mathrm{Eu}^{2+}$ and $\mathrm{Ce}^{3+}$. Here, the emission is from to $5 \mathrm{~d}-4 \mathrm{f}$ optical transitions. As electrons participate in the chemical bonding, the $\mathrm{d}-\mathrm{f}$ emission spectra consist of broad bands. These transition types are allowed and are consequently very fast (a few $\mu$ s or less). 
Some line emission is not a specific property of RE ions and in addition to that, in the case of RE ions, broad emission spectra can be obtained as well, depending on the optical transitions involved. In this respect, RE ions are not unique. The unique properties of the RE ions originate from the fact that the spectral position of the emission lines is almost independent of the host lattice, in contrast to line emission generate by the emission of metal ions.

\subsubsection{Radiative transitions: Intensities of optical transitions}

The terms shown in equation (13) are time-dependent; thereby, they do not lead to stationary states of the system. This way, they are treated using time-dependent perturbation theory resulting in transitions between the states established by the static interactions. In luminescent devices the most important term is $V_{E F}$ which gives rise to the emission and absorption of photons from decay radiative of the RE ions. This involves the interaction between the electron charge and the electric field and, the interaction between the electron spin and the magnetic field.

The experimental data on spectra of RE ions show that the radiation is mostly electric dipole (ED) nature, though some cases of the magnetic dipole radiation are also observed. Since the optical transitions take place between levels of a particular $4 \mathrm{f}^{\mathrm{N}}$ configuration, the electric dipole radiation is forbidden in first order, because the electric dipole operator has uneven parity and the transition matrix element must have even parity (Laporte selection rules). Van Vleck [55] pointed out the dipole electric radiation can only occur because the $4 \mathrm{f}^{\mathrm{N}}$ states have admixtures of $4 \mathrm{f}^{\mathrm{N}-1} \mathrm{nl}$ configuration ( $\mathrm{nl}$ will be mostly $5 \mathrm{~d}$ ), thus the $4 \mathrm{f}^{\mathrm{N}-1} \mathrm{nl}$ has to be chosen such that it has opposite parity from $4 \mathrm{f}^{\mathrm{N}}$. The admixture is produced via interactios that have odd parity and this depend of the host matrix of the RE ions. However, we can have four dominant sources of optical radiation in RE ions spectra:

i. Forced electric dipole induced by odd terms of the crystal field.

ii. Forced electric dipole radiation induiced by lattices radiation.

iii. Allowed magnetic dipole transition.

iv. Allowed electric quadrupole radiation.

In free atoms, magnetic dipole is about six orders of magnitude weaker than the electric dipole radiation. This latter occurs only as a consequence of a perturbation, both kinds of radiation shows up in the RE spectra with about intensity. Then, quadrupole radiation are less probable still in comparison with the magnetic dipole.

There are different types of transitions between levels (called electric dipole, electric quadrupole, and magnetic dipole) and therefore the transitions are divided into allowed ones (with a high probability) and so-called forbidden transitions (for transitions where the electric dipole transition is quantum mechanically forbidden).

For the first order allowed magnetic dipole radiation the free atom selection rules are still quite valid: $\Delta S=\Delta L=0$ and $\Delta J=0, \pm 1(0 \neq 0)$. In the electric dipole case we have: $\Delta l= \pm 1$, $\Delta S=0,|\Delta L|,|\Delta J| \leq 2 l$. However, since ED transitions is induced by the crystal field, the free 


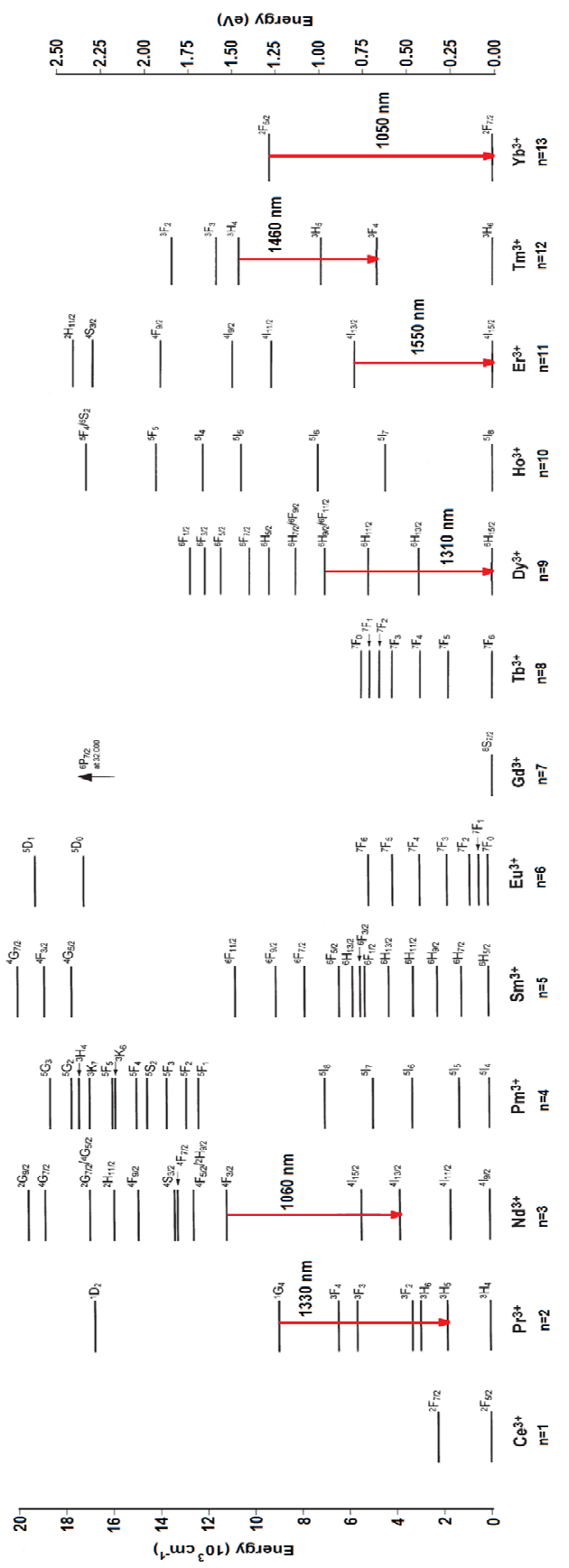

Figure 10. Energy levels of the triply charged lanthanide ions. Besides, the most technologically important radiative transitions are labeled. Figure adapted from A.J. Kenyon [47]. 
atom selection rules break down almost completely, i.e., the selection rules with regard to the $L, S$ and $J$ quantum numbers are now essential governed by the crystal field interaction yielding $|\Delta J| \leq 6$ (approximately). For the electric quadrupole case the rules selection is: $\Delta S=0,|\Delta L|,|\Delta J| \leq 2$.

We now outline the Judd-Ofelt theory for the determination of the intensities in RE crystal spectra [56,57]. It is in essence a quantification of the ideas formulated by van Vleck [55] under optical radiation sources. Intensities are often expressed in terms of oscillator strengths, where the total oscillator strength for a transition from an energy level $a$ to an energy level $b$ is given:

$$
f(a, b)=\frac{8 \pi^{2} m v}{3 h(2 J+1)}\left[\frac{\left(n^{2}+2\right)^{2}}{9 n} S_{e d}+n S_{m d}\right]
$$

Here $h$ is the Planck constant, $v$ the frequency of the transition $a \rightarrow b, n$ the refraction index of host. $S_{e d}$ and $S_{m d}$ are the electric dipole and magnetic dipole intensities respectively and are defined as:

$$
\begin{aligned}
& S_{e d}\left(\alpha J, \alpha^{\prime} J^{\prime}\right)=\sum_{\lambda=2,4,6} \Omega_{\lambda}\left\langle f^{N} \alpha[S L] J\left\|U^{(\lambda)}\right\| f^{N} \alpha^{\prime}\left[S^{\prime} L^{\prime}\right] J^{\prime}\right\rangle^{2} \\
& S_{m d}\left(\alpha J, \alpha^{\prime} J^{\prime}\right)=\beta^{2} \sum_{\lambda=2,4,6} \Omega_{\lambda}\left\langle f^{N} \alpha[S L] J|| L+2 S \| f^{N} \alpha^{\prime}\left[S^{\prime} L^{\prime}\right] J^{\prime}\right\rangle^{2}
\end{aligned}
$$

$U^{(\lambda)}$ is a tensor operator of rank $\lambda$, and the sum runs over the three terms values 2,4 and 6 of $\lambda$. With:

$$
\Omega_{\lambda}=(2 \lambda+1) \sum_{k, q} \frac{\left|B_{k, q}^{*}\right|^{2}\left|Y_{k, \lambda}\right|^{2}}{2 k+1}
$$

The $\Omega_{\lambda}$ parameters have so far been assumed to arise solely from crystal field; however, they also contain contributions from admixtures by the lattice vibrations. The asterisk means that for $B_{k, q}{ }^{*}$ the radial integral is to be taken between states $4 \mathrm{f}$ and $\mathrm{nl}$ instead of $4 \mathrm{f}$ and $4 \mathrm{f}$.

Also, it is interesting to determine the coefficient for spontaneous light from state $a(\alpha J)$ to state $b\left(\alpha^{\prime} J^{\prime}\right)$. Those are defined as:

$$
A\left(\alpha J, \alpha^{\prime} J^{\prime}\right)=A_{e d}+A_{m d}=\frac{64 \pi^{4} v^{3}}{3 h c^{2}(2 J+1)}\left[\frac{n\left(n^{2}+2\right)^{2}}{9} S_{e d}+n^{3} S_{m d}\right]
$$

Here $c$ velocity of light in vacuum. The Judd-Ofelt formalism has been applied to the analysis of a number of systems. In most of these analyses the crystal field splitting of the 
terms is neglected; therefore, the total absorption intensities between the ground term and the excited terms are analyzed with only three empirical parameters $\Omega_{\lambda}(\lambda=2,4$ and 6$)$.

If $b$ is an excited state that decays only by the emission of photons, its observed that relaxation rate is the sum of the probabilities for transitions to all possible final states. The total rate is the reciprocal of the excited-state lifetime $\tau_{a}$

$$
\tau_{a}=\frac{1}{A\left(\alpha J, \alpha^{\prime} J^{\prime}\right)}
$$

The branching ratio $\beta a, b$, for the transition $b \rightarrow a$ is the fraction of all spontaneous decay processes that occur through that channel and is defined as follows:

$$
\beta_{a, b}=\frac{A(a, b)}{\sum_{c} A(a, c)}=A(a, b) \tau_{a}
$$

The branching ratio, which has an important influence on the performance of a device based on a particular transition, appears often in the discussion of specific ions. It has a significant effect on the threshold of a laser and the efficiency of an amplifier.

\section{Metallic nanoparticle embedded in a gain media: LSPR interaction radiative transitions}

The RE-doped laser crystals and glasses are among the most popular solid-state gain media. In order of maintaining the efficiency of these materials in which the RE dopant is uniformily dispersed, as in multi-component glasses. In this sense, glasses have good optical, mechanical, and thermal properties to withstand the severe operating conditions of optical amplifier. Desirable properties include hardness, chemical inertness, absence of internal strain and refractive index variations, resistance to radiation-induced color centers, and ease of fabrication. Furthermore, the matrix host composition affects the solubility of the RE dopant, this affect the lifetime, absorption, emission, and excited state absorption cross sections of the dopant transitions [47]. This competing absorption phenomenon can seriously diminish the efficiency of an optical device. For example, a decrease in the excited-state absorption for $\mathrm{Er}^{3+}-$ doped fibers going from a germane-silicate host to an alumina-silicate host has been verified [58], demonstrating the importance of host selection for a given RE ion or laser transition. Host glasses compatible with this relatively high concentration of $\mathrm{RE}$ without clustering require the open, chain-like structure of phosphate glasses or the addition of modifier ions $(\mathrm{Ca}, \mathrm{Na}, \mathrm{K}, \mathrm{Li}$, or other) to open the silicate structure and increase solubility [48]. The limitation owing to clustering in a predominantly silica host without modifier ions has been well documented [19]. The maximum erbium concentration in silica for optimum amplifier performance has been suggested to be lesser to 100 ppm [59]. However a 14.4-dB gain, 900-ppm erbium-doped silica fiber amplifier has been reported, indicating that higher concentrations can produce useful devices [48]. Nevertheless, the interactions between host and RE ions, it is necessary to consider background losses from impurity absorption and scattering mechanisms that 
decrease the efficiency of the optical device. For example, depending on the phonon energies of the host matrix, some of the level lifetimes can be strongly quenched by multi-phonon transitions. Such effects are minimized in low-phonon-energy host media such as fluoride fibers. The effect of internal loss is most dramatic in distributed amplifiers, where pump light must travel long distances in the process of distributing gain.

Additionally, into the glass matrix can happen diverse kinds of interactions, in particular dipole-dipole interactions between the RE ions similar or of different species, allow energy transfer between those. This is exploited e.g. in $\mathrm{Er}^{3+}-\mathrm{Yb}^{3+}$-codoped fibers, where the pump radiation is dominantly absorbed by $\mathrm{Yb}^{3+}$ ions and mostly transferred to $\mathrm{Er}^{3+}$ ions [48].

In this way, RE-doped glasses shown are excellent materials that provide gain media. This makes them ideal candidates for embedding metallic NPs, resulting in new materials for the fabrication of devices optical with large enhancements even when the gain is saturated due light localization effects. It has been shown theoretically and experimentally that homogeneous aggregates of structures supporting LSPR can lead to extremely large enhancement of local field amplitudes exceeding those of single structures [60,61]. Besides, inorganic glasses are the host matrix for metallic NPs formation. The wide temperature range of glass viscosity growth provides the possibility to control over the NPs size within the wide range by means of modifying the temperature and duration of thermal processing. In fact, only such kind of matrix makes it possible to control and investigate all the stages of NPs formation, including the starting stage [14,62].

The LSPR in metallic NPs is predicted to exhibit a singularity when the surrounding dielectric medium has a critical value of optical gain [63]. This singularity is obtained from the equation (4), for $\operatorname{Re}\left[\varepsilon_{N P}(\omega)\right] \approx-2 \varepsilon_{m}$, the NP absorption presents a maximum. This is socalled the Frohlich condition is associated with dipole mode [1].

Therefore, the strong local electric field induced by the NP (LSPR) can enhance the total electric field, and can also improve the quantum yield of the luminenescence of the RE ions from the NP (this strong enhancement of electromagnetic fields is essential in nonlinear optics effects) [64,65]. Thus, the enhancement obtained with these NPs is due to the formation of EDs which generates a polarization given by $\vec{P}_{N P}=q e N^{\prime} \vec{x}$, where $\vec{x}$ is the distance between each NP, $q=\pi d^{3} / 6$ is the NP specific volume, $N^{\prime}$ is the conduction electrons density, and $e$ is the electronic charge [12]. Therefore, we obtained a modification in the local electric field by these dipoles (local field correction). Using the results obtained by O.L. Malta et al [66], the effective electric field can be written as:

$$
\vec{E}_{e f f}=\left(\varepsilon_{0}+2\right)\left[1+q \omega_{P}^{2} /\left\{3 \varepsilon_{0}\left[(1-q)\left(\omega_{P}^{2} / 3 \varepsilon_{0}\right)-\omega^{2}+i \gamma \omega\right]\right\}\right] \vec{E}_{0} / 3
$$

Where $\varepsilon_{0}$ is the dielectric constant in the presence of an external electromagnetic field of amplitude $E_{0}$. In the presence of an electromagnetic wave we have LSPR which forms EDs separated by different distance $\vec{r}$, some of which will contribute to the luminescence enhancement. 
Assuming that the RE ions may occupy different sites in the host, a direct coupling between the excited states of the RE ions and the NPs modifies the Stark levels energies [12,13,67]. As the NPs just give a contribution to the local field when the light is present, the oscillator strength of a spectral line, corresponding to transition from the ion ground level $i$ to the component $f$ of the excited level can be re-write as:

$$
f(a, b)=\frac{8 \pi^{2} m v}{3 h(2 J+1)}\left[\frac{\left(n^{2}+2\right)^{2}}{9 n} S_{e d}+\sum_{\lambda}\left|\left\langle i\left|D_{\lambda N P}^{(1)}\right| f\right\rangle\right|^{2}+n S_{m d}\right]
$$

The second term into parenthesis is added to the equation from the Judd-Ofelt theory [56,57], and represents the ED transition due to LSPR of NP. To obtain non vanishing matrix elements of the components $D_{\lambda N P}^{(1)}$ it is necessary to admix into $\langle i|$ and $|f\rangle$ other states of opposite parity.

Thus, the initial and final states are: $\langle i|=\left\langle\phi_{i}\right|+\sum_{\beta}\left\langle\phi_{i}|V| \phi_{\beta}\right\rangle /\left(E_{i}-E_{\beta}\right)\left\langle\phi_{\beta}\right|$, and

$$
\begin{gathered}
|f\rangle=\left|\phi_{f}\right\rangle+\sum_{\beta}\left\langle\phi_{\beta}|V| \phi_{f}\right\rangle /\left(E_{f}-E_{\beta}\right)\left|\phi_{\beta}\right\rangle \text {, then: } \\
\left\langle i\left|\vec{P}+\vec{P}_{N P}\right| f\right\rangle=\sum_{\beta}\left\{\frac{\left\langle\phi_{i}|V| \phi_{\beta}\right\rangle\left\langle\phi_{\beta}|\vec{P}| \phi_{f}\right\rangle}{E_{i}-E_{\beta}}+\frac{\left\langle\phi_{i}|\vec{P}| \phi_{\beta}\right\rangle\left\langle\phi_{\beta}|V| \phi_{f}\right\rangle}{E_{f}-E_{\beta}}+\frac{\left\langle\phi_{i}|V| \phi_{\beta}\right\rangle\left\langle\phi_{\beta}\left|\vec{P}_{N P}\right| \phi_{f}\right\rangle}{E_{i}-E_{\beta}} \ldots\right. \\
\left.\ldots+\frac{\left\langle\phi_{i}\left|\vec{P}_{N P}\right| \phi_{\beta}\right\rangle\left\langle\phi_{\beta}|V| \phi_{f}\right\rangle}{E_{f}-E_{\beta}}\right\}
\end{gathered}
$$

Where $V$ is the crystalline field, equation (16), responsible for the Stark levels and treated as a perturbation, $\phi_{i}$ and $\phi_{f}$ have the same parity, $\phi_{\beta}$ has opposite parity in relation of $\phi_{i}$ and $\phi_{f}$, $\vec{P}$ is the ED from the light interaction. For example, $\mathrm{Er}^{3+}$ ions presented an electronic configuration $[\mathrm{Xe}] 4 \mathrm{f}^{11}$, with $J=15 / 2$ been the spin-orbit ground state because the $\mathrm{f}$ shell is more than half-filled. The order of these Stark levels depends on the strength of the crystal field and the influence of $\vec{P}_{N P}$.

This can be observed experimentally in the luminescence spectra of different RE ions shown in Figure 11 interaction with silver or gold NPs in different host matrix, as a blue- or redshift (see equation 26) and a pronounced increase in luminescence (see equation 25).

In this scenario, the influence of $\vec{P}_{N P}$ appeared in the luminescence spectra as a blue or a red -shift of the peaks, e.g. a transfer energy from the NPs dipoles to RE ions, thus modifying the Stark energy level's bandwidth, see Figure 11. This energy transfer is obtained from the second term added in equation (25) and can be understood through the following definition: $D_{q^{\prime} N P}^{(1)}=\sum_{m} r_{m} C_{\lambda N P}^{(1)}\left(\theta_{m}, \phi_{m}\right)$, where $C_{\lambda N P}^{(1)}\left(\theta_{m}, \phi_{m}\right)$ is the Racah tensor and where $r_{m}$ is the distance between the NPs and the REions $\left(r_{m}=|\vec{x}|\right)$. 


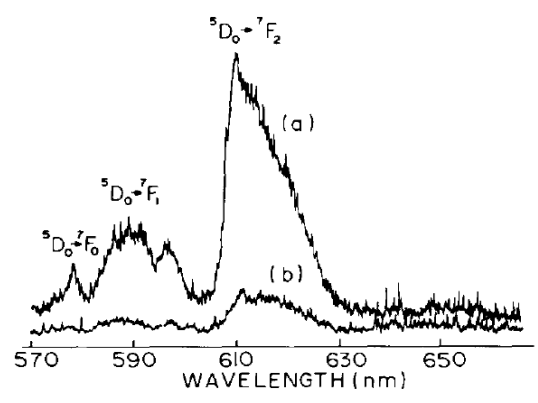

(a)
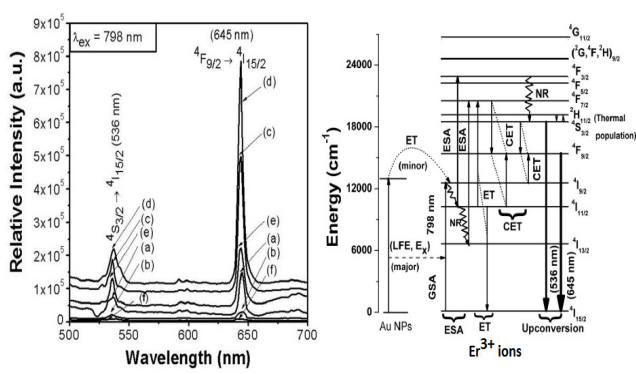

(c)

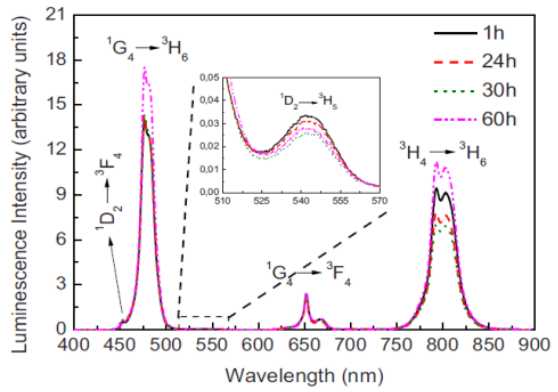

(e)

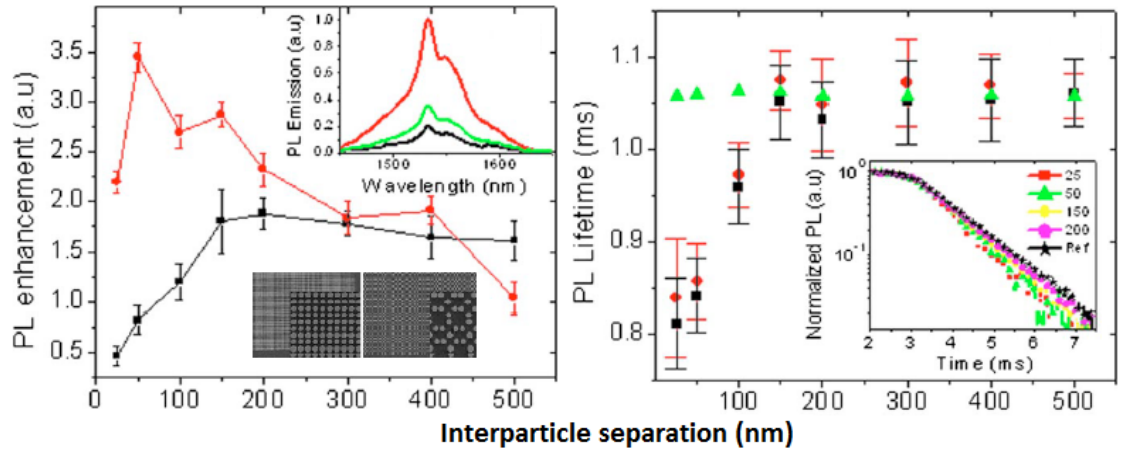

(g)

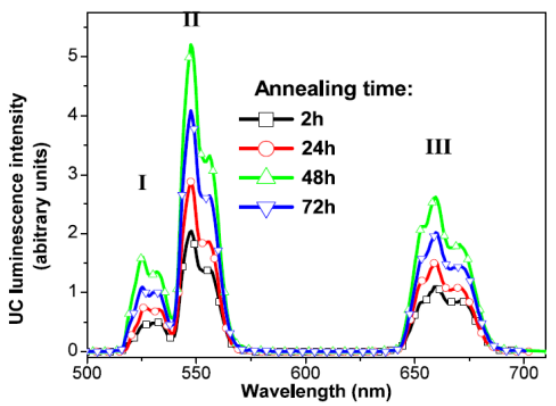

(b)

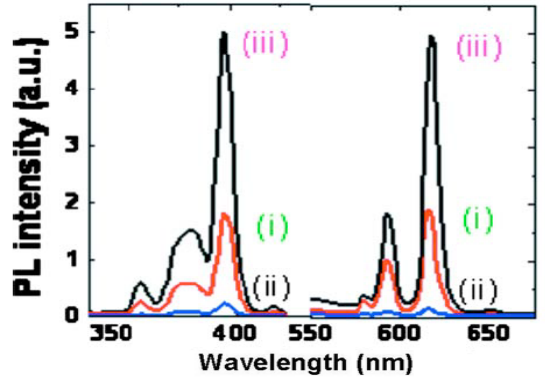

(d)

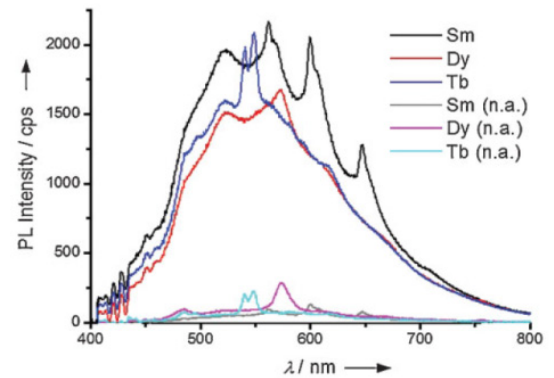

(f) 

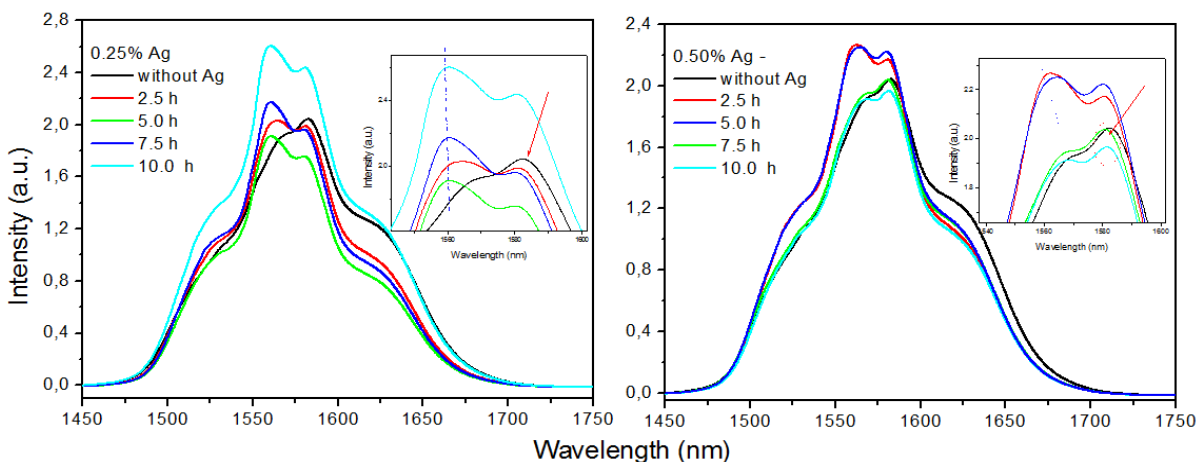

(h)
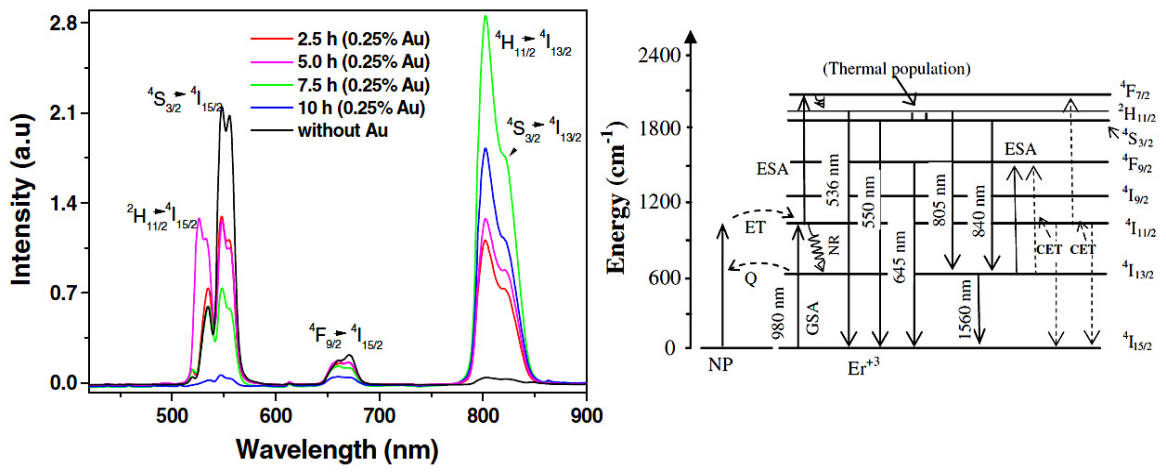

(i)

Figure 11. (a). Fluorescence spectra of the $\mathrm{Eu}^{3+}$ ions in the presence (a) and in the absence (b) of $\mathrm{Ag}$ NPs. The silver concentration is 7.5 in weight percent. The matrix glass composition can be found in [66]. (b). Frequency UC spectra for excitation at $980 \mathrm{~nm}$, (for composition see Ref. [68]. After the cooling the samples were annealed for different durations $(24,48$, and $72 \mathrm{~h}$ ) in order to nucleate silver NPs [68]. (c). Upconversion spectra extracted from [69] under excitation wavelength at $\lambda_{\mathrm{ex}}=798 \mathrm{~nm}$ (for composition and for amplification ratio see Ref. [69]. The bases of the emission curves (c), (d), and (e) have been uplifted for better visibility.

(d). PL spectra extracted from [70] (i) Eu(III)EDTA, 3 $\mathrm{H}_{2} \mathrm{O}$ complex, (ii) Eu complex with Au nanoparticles and (iii) Eu complex with $\mathrm{Au}-\mathrm{ZnO}$ nanoparticles.

(e). Upconversion spectra of $\mathrm{Tm}^{3+} / \mathrm{Yb}^{3+}$ codoped $\mathrm{PbO}-\mathrm{GeO}_{2}$ samples containing silver NPs obtained by pumping the samples with a diode laser operating at $980 \mathrm{~nm}$. For more details see [71].

(f). PL spectra $\left(\lambda_{\text {exc }} \approx 337 \mathrm{~nm}\right)$ of sy-activated with $0.037 \mathrm{~mol} \% \mathrm{Ag}$ containing glasses co-doped with 0.19 mol\% Sm (black curve), Dy (red), and Tb (blue), respectively, and of non-activated and non-annealed (n.a.) $0.037 \mathrm{~mol} \% \mathrm{Ag}$ containing samples co-doped with $0.19 \mathrm{~mol} \% \mathrm{Sm}$ (grey), Dy (magenta), and Tb (light blue) [72].

(g). Picture on the left, Integrated PL enhancement in periodic (circle) and Fibonacci (square) nanoparticle arrays of various interparticle separations. The top inset shows the representative PL spectrum of periodic (bottom), Fibonacci (top) nanoparticle arrays with $\Delta \min =50 \mathrm{~nm}$ and unpatterned area (middle), and the inset bottom shown the SEM micrograph of (left) periodic, (right) Fibonacci array Au nanocylinders. Picture on the right, PL lifetime of periodic (circle), Fibonacci (square), unpatterned 
(triangle). Inset Er decay of unpatterned (star) and Fibonacci arrays with different $\Delta$ min as specified in the legend. For more details see Ref. [73].

(h). Picture on the left; PL of the TE025-Y samples pumped with diode laser at $980 \mathrm{~nm}$, showing PL enhancement. The inserted figure shows a zoom of the peaks. The vertical dashed line is a reference for showing the blue-shift of the peaks, and the arrow indicates the enhancement due to transfer energy from EDs to $\mathrm{Er}^{3+}$. Picture on the right; same for the samples TE050-Y. The enhancement of luminescence was found to be reproducible for all ours samples. These pictures was extracted and modified from Ref. [12]. These spectrums are of $\mathrm{Er}^{3+}$-doped tellurite glass, more details see [12].

(i). Luminescence spectra of $\mathrm{Er}^{3+}: \mathrm{Au}$-doped tellurite glass for several annealing times, pumped at 980 $\mathrm{nm}$. Picture on the left; Upconversion, for the range $400<\lambda<900 \mathrm{~nm}$. Picture on the right; Energy-levels diagram of $\mathrm{Er}^{3+}$ for the luminescence spectra. ET stands for energy transfer, CET for cooperative energy transfer, Q for quenching, GSA for ground state absorption, NR for non-radiative decay, and ESA for excited state absorption. For more details see Ref. [13].

Nevertheless, an interesting question arises: how these NPs are excited?

As explained above, those NPs can be excited by a predefined incident radiation through a direct coupling between the excited states of the both RE ions and NPs, resulting in: (i) a local field increase (Frohlich condition), at $\omega_{p}$, (ii) a nonradiatively decay (heat generation by Joule effect) or (iii) a radiative energy release which depends on the albedo of the NPs. Thereby, the exact response of LSPR will depend on the details of the physical system (e.g. arrangement, shape, host matrix), and usually not strictly symmetric about the resonant frequency. A schematic representation of the interaction process within the RE:NP system is depicted in Figure 12 (a).

Also we can elucidate the enhancement (quenching) from the process of energy transfer as following: $\mathrm{Er}^{3+}$ emission promotes energy transfers into a plasmonic mode, which can decay nonradiatively by heat generation (Joule effect) or radiatively by releasing energy that depends on the albedo of the NPs. We consider two types of emission from a system of identical dipoles [74]:

$$
\begin{gathered}
I_{1} \propto I_{p} \eta_{p} \eta_{0} \\
I_{2} \propto I_{1} \eta_{0} \eta_{L S P R} Q_{\text {scatt }}
\end{gathered}
$$

Here, $I_{1}$ is the intensity emission for a single emitter, $I_{2}$ is the intensity emission of the NP, $I_{p}$ the pump intensity, $\eta_{p}$ is the pump efficiency, $\eta_{0}$ is the internal quantum efficiency of the energy transfer, $\eta_{L S P R}$ is the efficiency of the energy transfer (nonradiative) to LSPR modes and the scattering efficiency of the plasmon at the emission wavelength $Q_{\text {scatt }}$ is:

$$
Q_{\text {scatt }}=Q_{\text {scatt }}^{(\text {Ray })}=\frac{8}{3} \frac{\omega_{p}^{4} y^{4}}{\left(\omega^{2}-\omega_{p}^{2}\right)^{2}+\frac{4}{9} y^{6} \omega_{p}^{4}}
$$

Where $Q_{\text {scatt }}^{(\text {Ray })}$ is the Rayleigh scattering $[67,68]$ and, $y=\omega D / c$, with $c$ the speed of light. Therefore the total intensity $I_{T}$, can be written as [62]: 


$$
I_{T}=I_{1}\left(1-\eta_{L S P R}\right)+\eta_{0} I_{2}
$$

We consider $I_{1}$ as the contribution from the emitter dipole (RE ions) uncoupled to the metal and $I_{2}$ the plasmon enhanced emission from coupled dipoles, see Figure 12 (b).

Finally, we can obtain the following conditions: $\eta_{0}{ }^{2} Q_{\text {scatt }}^{(\text {Ray })} \geq 1$, where $Q_{\text {scatt }}^{(\text {Ray })}(\omega, D)$, i.e. the luminescence enhancement depends on the incident radiation frequency and the NP size. But, as were mentioned, the incident radiation for the NP activation comes from the emitter dipole $\left(I_{1} \eta_{L S P R}\right)$ and not from the pump radiation $\left(I_{p}\right)$, i.e., from transition radiative from RE. The coupling efficiency $\eta_{L S P R}$ is defined by: $\eta_{L S P R}=\Gamma_{L S P R} /\left(\Gamma_{\text {rad }}+\Gamma_{\text {nonrad }}+\Gamma_{L S P R}\right)$, where: $\Gamma_{L S P R}$ is the energy transfer rate to the LSPR mode $[73,74]$.

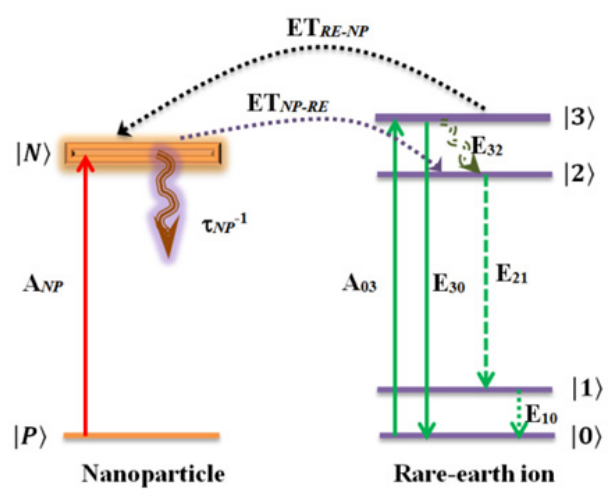

(a)

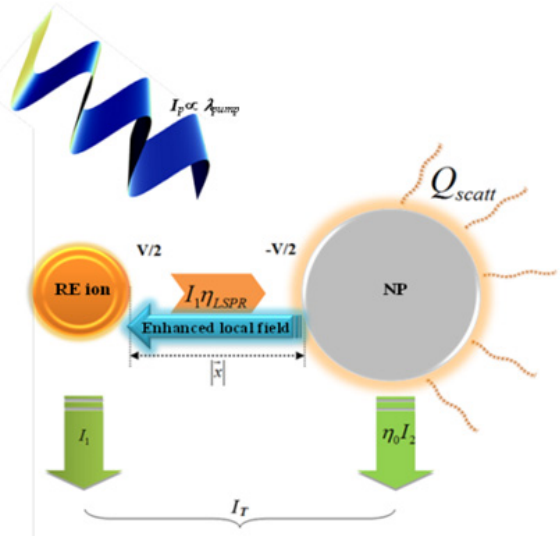

(b)

Figure 12. (a) Energy level scheme for a resonant and RE ion absorption. The dot line indicates the energy transfer between $\mathrm{RE} \rightarrow \mathrm{NP}$ or $\mathrm{NP} \rightarrow \mathrm{RE}$, and the vertical dot line shows the transition radiative under consideration. The curved arrows indicate non-radiative transitions. (b) Schematic representation of the system RE:NP. A monochromatic plane wave with pump intensity $\left(I_{p}\right)$ which is proportional to the pump wavelength $\lambda_{\text {pump, }}$ induces the following processes: (i) absorption of RE ion, $I_{p}$, (ii) activation of NPs, due the coupling RE:NP (transitions levels), $I_{1} \eta_{L S P R}$ (iii) NP transmitter, via electric dipole. Such coupling depends on the coupling efficiency $\eta_{L S P R}$. Where $I_{1}$ the intensity emission of emitter, $I_{2}$ the intensity emission of the NP, $\eta_{L S P R}$ the efficiency of the energy transfer (nonradiative) to LSPR modes. Equipotential surfaces (electric dipole coupling) with electric potentials $-\mathrm{V} / 2$ and V/2 for the NPs and the $\mathrm{Er}^{3+}$ ions respectively.

The strong local electric field induced by NPs (ED), increases the quantum yield $\eta$ of the RE luminescence, defined by the ratio of the local field $\vec{E}_{l o c}$ and the incident field $\vec{E}_{i}$, $\eta=\left(r_{m}+d\right) / d=\left|\vec{E}_{l o c}\right| /\left|\vec{E}_{i}\right|[14]$, here $\vec{E}_{\text {eff }}=\vec{E}_{l o c}+\vec{E}_{i}$. Thus, the maximum field enhancement is determined by the shortest distance between two equipotential particles. It corroborates well with the demonstration in the references $[12,13,67,75]$.

Another possible mechanism for the energy transfer is due to that part of silver or gold (small silver or gold aggregates) probably remained under the form of ions, atoms, charged 
or neutral dimmers and multimers. Consequently, the contribution of the latter species to the RE luminescence enhancement and band shape features cannot be excluded [14], Figure 11. Moreover, the insertion of silver or gold in the samples leads to strong modification of the glassy network and consequently on the RE local environment, this is verified in reference [62].

\section{Conclusion}

This chapter present results where it was demonstrated the simultaneous exploitation of the enhanced local field due to NPs and energy transfer processes in order to enhance the luminescence spectra of a glassy composite material, and others. Besides, from these nanoparticles it is possible to modulate the down/up-conversion emission of the REs with applicability in areas such as optical telecommunication, including biomedical imaging and energy conversion. On the other hand, this significant enhanced fluorescence has high potential for application in photonics, optical displays, lasers and optical memory devices, amongst others. However, the success of new applications of nanoparticles depends on improvement in the understanding of the properties of LSPR and the environment around them. Further efforts and systematic studies must be realized in order to offer new developments to extend the analytical applications field of metallic nanoparticle interaction with rare-earth ions.

We hope that this chapter stimulated our readers for the development of theoretical and experimental work on plasmonics and rare-earths.

\section{Author details}

V.A.G. Rivera, F.A. Ferri and E. Marega Jr.

Instituto de Física de São Carlos, INOF/CEPOF, USP, São Carlos - São Paulo, Brazil

\section{Acknowledgement}

This work was financially supported by the Brazilian agencies FAPESP, CNPq and CEPOF/INOF. V.A.G. Rivera thanks to FAPESP for financial support (project 2009/08978-4 and 2011/21293-0) that allowed my pos-doctoral and my gratefully to Dr. Luiz Antonio Nunes of the Instituto de Fisica de São Carlos - University São Paulo - Brazil and the Dr. Yannick Ledemi and the Dr. Younnes Messaddeq of the Centre d'Optique, Photonique et laser - University Laval- Canada for the discussions on this issue.

\section{References}

[1] Stefan Alexander Maier. Plasmonics Funadamentals and Applications. Springer Science+Business Media LLC; 2007.

[2] Mark I. Stockman. Opt. Exp. 2011;19(22): 22029-22106.

[3] Y. Wang, E. W. Plummer and K. Kempa. Advances in Physics 2011;60(5): 799-898. 
[4] K. Kneipp, H. Kneipp, I. Itzkan, R. R. Dasari, and M. S. Feld. J. Phys.: Condens. Matter 2002;14:R597-R624.

[5] Y. Takeda and N. Kishimoto. Nuc. Inst. Met. Phys. Res. B 2003;206: 620-623.

[6] Peter J. Reece. Nat. Phot. 2008;2:333-334.

[7] Desurvire E., J. L. Zyskind and C. R. Giles. IEEE/OSA J. Ligh. Technol. 1990;LT8: 17301741.

[8] A. N. Sudarkin and P. A. Demkovich. Sov. Phys. Tech. Phys. 1989;34: 764-766.

[9] I. Avrutsky. Phys. Rev. B 2004;70: 155416-155421.

[10] M. P. Nezhad, K. Tetz, and Y. Fainman. Opt. Express 2004;12: 4072-4079.

[11] Chau, K. J., Dice, G. D., and Elezzabi, A. Y. Phys. Rev. Lett. 2005;94: 173904-173907.

[12] V.A.G. Rivera, S. P. A. Osorio, Y. Ledemi, D. Manzani, Y. Messaddeq, L. A. O. Nunes, and E. Marega. Opt. Exp. 2010;18: 25321-25328.

[13] S.P.A. Osorio, V.A.G. Rivera, L.A.O. Nunes, E. Marega, D. Manzani, Y. Messaddeq. Plasmonics 2012;7: 53-58.

[14] V.A.G. Rivera, Y. Ledemi, S.P.A. Osorio, D. Manzani, Y. Messaddeq, L.A.O. Nunes and E. Marega. J. Non-Crys. Sol. 2012;358: 399-405.

[15] X. Lu, M. Rycenga, S.E. Skrabalak, B.Wiley, and Y. Xia. Annu. Rev. Phys. Chem. 2009;60: 167-192.

[16] U. Kreibig, M. Vollmer. Optical Properties of Metal Clusters. Springer-Verlag, Berlin; 1995.

[17] Jackson John D. Classical Electrodynamics. John Wiley \& Sons, Inc., New York, NY, 3rd edition; 1999.

[18] Bohren, Craig F. and Huffman Donald R. Absorption and scattering of light by small particles. John Wiley \& Sons, Inc., New York, NY, 1 edition; 1983.

[19] Mie Gustav. Ann. Phys. 1908;25: 377-345.

[20] E. D. Palik. Handbook of Optical Constants of Solids Academic. Elsevier, Orlando, FL. ISBN: 978-0-12-544415-6; 1985.

[21] K. Lance Kelly, Eduardo Coronado, Lin Lin Zhao, and George C. Schatz. J. Phys. Chem. B 2003;107: 668-677.

[22] Novotny L. and Hecht B. Principles of nano-optics, Cambridge University Press, United Kingdom, ISBN-13 968-0-521-83224-3; 2006.

[23] Hideki Nabika and Shigehito Deki. J. Phys. Chem. B 2003;107(35): 9161-9164.

[24] D. D. Evanoff, R. L. White and G. Chumanov. J. Phys. Chem. B 2004;108(37): 1522-1524.

[25] H. Baida, P. Billaud, S. Marhaba, D. Christofilos, E. Cottancin, A. Crut, J. Lermé, P. Maioli, M. Pellarin, M. Broyer, N. Del Fatti, and F. Vallé. Nano Lett. 2009;9(10): 34633469.

[26] C. Voisin, N. Del Fatti, D. Christofilos and F.J. Valleé. Phys. Chem. B 2001;105: 22642280.

[27] F. Hache, D. Ricard and C.J. Flytzanis. J. Opt. Soc. Am. B 1986;3(12): 1647-1655.

[28] S. Link, M. B. Mohamed and M. A. El-Sayed. J. Phys. Chem. B 1999;103: 3073-3077.

[29] H. Kuwata, H. Tamaru, K. Esumi and K. Miyano. Appl. Phys. Lett. 2003;83: 4625-4627.

[30] S.W. Prescott and P. Mulvaney. J. Appl. Phys. 2006;99: 123504-123510. 
[31] Cheng-ping Huang, Xiao-gang Yin, Huang Huang and Yong-yuan Zhu. Opt. Exp. 2009;17(8): 6407-6413.

[32] Mock J. J., Barbic M., Smith D. R., Schultz D. A., and Schultz S. J. Chem. Phys. 2002;116(15): 6755-6759.

[33] Purcell E. M. and Pennypacker C. R. Astrophys. 1973;186: 705-714.

[34] Ruppin R. Phys. Rev. B 1982;26: 3440-3444.

[35] Pinchuk A., Kalsin A., Kowalczuk B., Schatz G. and Grzybowski B. J. Phys. Chem. C 2007;111: 11816-11822.

[36] Gerardy J. M. and Ausloos M. Phys. Rev. B 1982;25: 4204-4229.

[37] Claro F. Phys. Rev. B 1984;30: 4989-4999.

[38] Rojas R. and Claro F. Phys. Rev. B 1986;34: 3730-3736.

[39] Olivares I., Rojas R., Claro F. Phys. Rev. B 1987;35: 2453-2455.

[40] M. Chergui, A. Melikian , H. Minassian. J. Phys. Chem. C 2009;113: 6463-6471.

[41] P. P. Sorokin and M. J. Stevenson. Phys. Rev. Lett. 1960;5: 557-559.

[42] D.C. Brown. IEEE J. Sel. Top. Quantum Electron. 2005;11: 587-599.

[43] S. Hufner. Optical Spectra of Transparent Rare Earth Compounds. Academic press New York - San Francisco - London; 1978.

[44] B.R. Judd. Operator Techniques in Atomic Spectroscopy. McGraw-Hill, New York; 1963.

[45] B.G. Wybourne. Spectroscopic properties of Rare Earths, Wiley, New York; 1965.

[46] A.J. Freeman and R.E. Watson. Phys. Rev. 1962;127: 2058-2075.

[47] A.J. Kenyon. Prog. Quan. Elec. 2002;26: 225-284

[48] Michel J.F. Digonnet and Marcel Dekker. Rare-earth-doped fiber lasers and amplifiers. 2d ed., edited by, Inc. New York - Basel; 2001.

[49] V.A.G. Rivera, E.F. Chillce, E.G. Rodrigues, C.L. Cesar, L.C. Barbosa. J. Non-Crys. Sol. 2006;353: 125-130.

[50] V.A.G. Rivera, E.F. Chillce, E.G. Rodrigues, C.L. Cesar, L.C. Barbosa. Proc. SPIE 2006;6116: 190-193.

[51] P.J. Mears, L. Reekie, I.M. Jauncey and D.N. Payne. Elec. Lett. 1987;23: 1026-1028.

[52] E. Desurvire, R.J. Simpson and P.C. Becker. Opt. Lett. 1987;12: 888-890.

[53] E. Desurvire. Phys. Today 1994;47: 20-27.

[54] M. Yamane and Y. Asahara. Glasses for Photonics. Cambridge - University Press, Cambridge, United Kingdom; 2002.

[55] J.H. van Vleck. J. Phys. Chem. 1937;41: 67-80.

[56] B.R. Judd. Phys. Rev. 1962;127: 750-761.

[57] G. S. Ofelt. J. Chem. Phys. 1962;37: 511-520.

[58] Arai K., H. Namikawa, K. Kumata, T. Honda, Y. Ishii, T. Handa. J. Appl. Phys. 1986;59: 3430-3436.

[59] Shimizu M., M. Yamada, M. Horigucho and E. Sugita. IEEE Pho. Tech. Lett. 1990;2: 4345.

[60] V.A. Markel, V. M. Shalaev, E. B. Stechel, W. Kim, and R. L. Armstrong, Phys. Rev. B 1996;53: 2425.

[61] V. M. Shalaev, E. Y. Poliakov and V. A. Markel. Phys. Rev. B 1996;53: 2425-2449. 
[62] V.A.G. Rivera, S.P.A. Osorio, D. Manzani, Y. Messaddeq, L.A.O. Nunes, E. Marega Jr. Opt. Mat. 2011;33: 888-892.

[63] N. M. Lawandy. Appl. Phys. Lett. 2004;85: 5040-5042.

[64] S. Kim, J. Jin, Y. Kim, I. Park and Y. Kim. Nature 2008;453: 757-760.

[65] S. Kuhn, U. Hakanson, L. Rogobete and V. Sandoghdar. Phys. Rev. Lett. 2006;97: 017402.

[66] O.L. Malta, P.O. Santa-Cruz, G.F. de Sá and F. Auzel. J. of Lumin. 1985;33: 261-272.

[67] T. Som and B. Karmakar. J. Appl. Phys. 2009;105: 013102.

[68] L.R.P. Kassab, F.A.Bonfim, J.R. Martinelli, N.U. Wetter, J.J.Neto and Cid B. Araujo. Appl. Phys. B 2009;94: 239-242.

[69] T. Som and B. karmakar. J. Opt. Soc. Am. B 2009;26(12): B21-B27.

[70] Krishna Kanta Haldar and Amitava Patra. App. Phys. Lett. 2009;95: 063103.

[71] Thiago A. A. Assumpção, Davinson M. da Silva, Luciana R. P. Kassab and Cid B. de Araújo. J. App. Phys. 2009;106: 063522.

[72] Maik Eichelbaum and Klaus Rademann. Adv. Funct. Mater. 2009;19: 2045-2052.

[73] A. Gopinath, S. V. Boriskina, S. Yerci, R. Li and L. Dal Negro. Appl. Phys. Lett. 2010;96: 071113.

[74] J. R. Lakowicz. Anal. Biochem. 2005;337: 171-194.

[75] F. Le, D. W. Brandl, Y. A. Urzhumov, H. Wang, J. Kundu, N. J. Halas, J. Aizpurua, and P. Nordlander. ACS Nano 2008;2: 707-718. 